\title{
ALGEBRAIC EXTENSIONS OF POWER SERIES RINGS
}

\author{
BY
}

\author{
JIMMY T. ARNOLD
}

\begin{abstract}
Let $D$ and $J$ be integral domains such that $D \subset J$ and $J[[X]]$ is not algebraic over $D[[X]]$. Is it necessarily the case that there exists an integral domain $R$ such that $D[[X]] \subset R \subseteq J[[X]]$ and $\left.R \cong D[[X]]\left[\left\{Y_{i}\right\}_{i=1}^{\infty}\right]\right]$ ? While the general question remains open, the question is answered affirmatively in a number of cases. For example, if $D$ satisfies any one of the conditions (1) $D$ is Noetherian, (2) $D$ is integrally closed, (3) the quotient field $K$ of $D$ is countably generated as a ring over $D$, or (4) $D$ has Krull dimension one, then an affirmative answer is given. Further, in the Noetherian case it is shown that $J[[X]]$ is algebraic over $D[[X]]$ if and only if it is integral over $D[[X]]$ and necessary and sufficient conditions are given on $D$ and $J$ in order that this occur. Finally if, for every positive integer $n$, $D\left[\left[X_{1}, \ldots, X_{n}\right]\right] \subset R \subseteq J\left[\left[X_{1}, \ldots, X_{n}\right]\right]$ implies that $R \approx$ $\left.D\left[\left[X_{1}, \ldots, X_{n}\right]\right]\left[\left\{Y_{i}\right\}_{i=1}^{\infty}\right]\right]$, then it is shown that $J\left[\left[X_{1}, \ldots, X_{n}\right]\right]$ is algebraic over $D\left[\left[X_{1}, \ldots, X_{n}\right]\right]$ for every $n$.
\end{abstract}

1. Introduction. Throughout this paper $D$ and $J$ denote integral domains (with identity) having quotient fields $K$ and $L$, respectively, and such that $D \subseteq J$. If $L=K$ we say that $J$ is an overring of $D$. In the case when $J=K$ Gilmer has shown in [2] that $J[[X]]$ is an overring of $D[[X]]$ if and only if $\cap_{i=1}^{\infty}\left(a_{i}\right) \neq(0)$ for each subset $\left\{a_{i}\right\}_{i=1}^{\infty}$ of nonzero elements of $D$. Sheldon has shown in [5] that if $a$ is a nonzero element of $D$ such that $\bigcap_{i=1}^{\infty}\left(a^{i}\right)=(0)$ and if $J=D[1 / a]$, then the quotient field of $J[[X]]$ has infinite transcendence degree over the quotient field of $D[[X]]$ (hereafter we will call this the transcendence degree of $J[[X]]$ over $D[[X]])$. Thus, if $J=D[1 / a]$ then either $J[[X]]$ is an overring of $D[[X]]$ or has infinite transcendence degree over $D[[X]]$. Motivated by these results, Arnold and Boyd asked in [1] whether this is the case for an arbitrary overring $J$ of $D$. A simple example is given in [1, Example 1.2] of a domain $D$ with characteristic $p \neq 0$ and an overring $J$ of $D$ such that $(J[[X]])^{p} \subseteq D[[X]]$ but $J[[X]]$ is not an overring of $D[[X]]$. Thus, if $D$ has characteristic $p \neq 0$ the appropriate question is whether either $J[[X]]$ has infinite transcendence degree over $D[[X]]$ or there exists a nonnegative integer $m$ such that $(J[[X]])^{p^{m}}$ is contained in the quotient field of $D[[X]]$. This led, in [1], to the consideration of the equivalence of statements (a)-(d) below. Statement (e) is included for future reference.

(1.1) In each of the following statements $J$ is an overring of $D, D^{*}=D \backslash(0)$, and $p$ is the characteristic of $D$ if $D$ has nonzero characteristic, while $p=1$ if $D$ has characteristic zero.

Received by the editors March 7, 1980 and, in revised form, June 24, 1980.

AMS (MOS) subject classifications (1970). Primary 13J05; Secondary 13G05, 13 B99.

Key words and phrases. Power series ring, algebraic extension, integral extension.

(C) 1981 American Mathematical Society $0002-9947 / 81 / 0000-0406 / \$ 05.00$ 
(a) For each subset $\left\{\xi_{i}\right\}_{i=1}^{\infty}$ of $J$ there exists a nonnegative integer $m$ and an element $d$ in $D^{*}$ such that $d \xi_{i}^{p^{m}} \in D$ for each $i$.

(b) There exists a nonnegative integer $m$ such that $(J[[X]])^{p^{m}} \subseteq(D[[X]])_{D^{*}}$.

(c) $J[[X]]$ is algebraic over $D[[X]]$.

(d) $J[[X]]$ has finite transcendence degree over $D[[X]]$.

(e) If $R$ is an integral domain such that $D[[X]] \subset R \subseteq J[[X]]$, then $R \cong$ $D[[X]]\left[\left[\left\{Y_{i}\right\}_{i=1}^{\infty}\right]\right]$ via a $D[[X]]$-isomorphism.

In general the implications $(\mathrm{a}) \leftrightarrow(\mathrm{b}) \rightarrow(\mathrm{c}) \rightarrow(\mathrm{d})$ hold [1, Proposition 2.1] and it is shown in [1] that conditions (a)-(d) are equivalent if $D$ is Noetherian [1, Theorem 2.5], if $D$ is root closed [1, Theorem 1.6], if $K$ is countably generated as a ring over $D$ [1, Theorem 2.4], or if $J$ is a quotient overring of $D$ [1, Theorem 1.10]. Whether (a)-(d) are equivalent in general is an open question. In this paper we wish to remove the assumption that $J$ is an overring of $D$ and, since clearly $(d) \rightarrow(e)$, we wish to strengthen the basic question asked in [1] to the following. Is it the case that either $J[[X]]$ is algebraic over $D[[X]]$ (in some "nice" way) or there exists an integral domain $R$ such that $D[[X]] \subset R \subseteq J[[X]]$ and $R \cong$ $D[[X]]\left[\left[\left\{Y_{i}\right\}_{i=1}^{\infty}\right]\right]$ ? To be more specific, we ask whether statements (1)-(5) below are equivalent.

(1.2) In each of the following statements $D^{*}=D \backslash(0), K_{0}$ is the maximal, separable extension of $K$ in $L$, and $p$ is the characteristic of $D$ if $D$ has nonzero characteristic while $p=1$ if $D$ has zero characteristic.

(1) $L$ is algebraic over $K,\left[K_{0}: K\right]$ is finite, and if $D_{0}$ is any integral domain such that $D \subseteq D_{0} \subseteq J$ and $D_{0}$ has quotient field containing $K_{0}$, then for each subset $\left\{\xi_{i}\right\}_{i=1}^{\infty}$ of $J$ there exists a nonnegative integer $m$ and a nonzero element $d$ in $D$ such that $d \xi_{i}^{p^{m}} \in D_{0}$ for each positive integer $i$.

(2) There exists a nonnegative integer $m$ and a finite integral extension $D_{0}=D[\theta]$ of $D$ such that $K_{0}=K(\theta), D_{0}$ has quotient field $K_{0}, D_{0} \subseteq J$, and $(J[[X]])^{p^{m}} \subseteq$ $\left(D_{0}[[X]]\right)_{D^{*}}$. If $K_{0}=K$ we can take $D=D_{0}$.

(3) $J[[X]]$ is algebraic over $D[[X]]$.

(4) $J[[X]]$ has finite transcendence degree over $D[[X]]$.

(5) If $R$ is an integral domain such that $D[[X]] \subset R \subseteq J[[X]]$ then $R \notin$ $D[[X]]\left[\left[\left\{Y_{i}\right\}_{i=1}^{\infty}\right]\right]$ via a $D[[X]]$-isomorphism.

$\S 2$ is devoted primarily to the proof of the key result of this paper, Theorem 2.1, which gives necessary conditions in order that (5) of (1.2) hold. In $\$ 3$ it is shown that $(1) \leftrightarrow(2) \rightarrow(3) \rightarrow(4) \rightarrow(5)$ and sufficient conditions are given on $D$ and $J$ in order that (1)-(5) be equivalent. In particular, (1)-(5) are shown to be equivalent if $D$ is Noetherian (Theorem 3.9), if $D$ is integrally closed (Theorem 3.10), if the quotient field $K$ of $D$ is countably generated as a ring over $D$ (Theorem 3.11 ), if $D$ has Krull dimension one (Theorem 3.12), or if $J$ is a quotient overring of some finite integral extension of $D$ (Theorem 3.14). In [3] Gilmer obtains necessary and sufficient conditions on the fields $K$ and $L$, with $K \subseteq L$, in order that $L[[X]]$ be integral over $K[[X]]$. Theorem 3.9 shows that if $D$ is Noetherian, then $J[[X]]$ is algebraic over $D[[X]]$ if and only if it is integral over $D[[X]]$ and the necessary and sufficient conditions established by Gilmer in [3] are extended to this case. The basic question remains open. 
(1.3) Open question. Are conditions (1)-(5) of (1.2) equivalent?

We conclude $\S 3$ by proving, in Theorem 3.17 , that $J\left[\left[X_{1}, \ldots, X_{n}\right]\right]$ is algebraic over $D\left[\left[X_{1}, \ldots, X_{n}\right]\right]$ for every positive integer $n$ if and only if, for every $n$, $D\left[\left[X_{1}, \ldots, X_{n}\right]\right] \subset R \subseteq J\left[\left[X_{1}, \ldots, X_{n}\right]\right]$ implies that

$$
R \approx D\left[\left[X_{1}, \ldots, X_{n}\right]\right]\left[\left[\left\{Y_{i}\right\}_{i=1}^{\infty}\right]\right] \text {. }
$$

Throughout the paper $D^{*}=D \backslash(0), K_{0}$ denotes the maximal, separable extension of $K$ in $L[6, \mathrm{p} .123]$, and $p$ denotes the characteristic of $D$ if $D$ has nonzero characteristic while $p=1$ if $D$ has characteristic zero. If $L / K$ is algebraic then $L / K_{0}$ is purely inseparable and we say that $L$ has finite exponent over $K_{0}$ if there exists a nonnegative integer $n$ such that $L^{p^{n}} \subseteq K_{0}[6$, p. 123].

For a commutative ring $R, R[[X]]$ denotes the power series ring over $R$. If $f=\sum_{i=0}^{\infty} r_{i} X^{i} \in R[[X]]$ then the order of $f$, denoted $o(f)$, is the smallest integer $n$ such that $r_{n} \neq 0$. If $I$ is an indexing set we write $Y_{I}$ (or $Y$ if $I$ is understood) to denote the set $\left\{Y_{i}\right\}_{i \in I}$ of analytic indeterminates over $R$ and $R\left[\left[Y_{I}\right]\right]$ denotes the full power series ring in these indeterminates. (In the notation of $[4, \mathrm{p} .10], R\left[\left[Y_{I}\right]\right]$ denotes the ring $R\left[\left[\left\{Y_{i}\right\}_{i \in I}\right]\right]_{3}$.) If $\Lambda(I)=\bigoplus_{i \in I} S_{i}$, where each $S_{i}$ is the additive semigroup of nonnegative integers, and if $\pi_{i}: \Lambda(I) \rightarrow S_{i}$ is the canonical projection, then for $\alpha \in \Lambda(I)$ we set $Y_{I}^{\alpha}=\prod_{\left\{i \in I \mid \pi_{i}(\alpha) \neq 0\right\}} Y_{i}^{\pi_{i}(\alpha)}$ and we write $f=\Sigma_{\alpha \in \Lambda(I)} r_{\alpha} Y^{\alpha}$ to denote an arbitrary element of $R\left[\left[Y_{I}\right]\right]$. We are interested only in the case when $I$ is countable. In this case suppose that $D \subset J$ and let $\mathscr{F}=\left\{f_{i}\right\}_{i \in I}$ be a subset of $X J[[X]]$ such that $\left\{f_{i} \in \mathscr{F} \mid o\left(f_{i}\right) \leqslant k\right\}$ is finite for each positive integer $k$. Then the correspondence $Y_{i} \rightarrow f_{i}$ determines a unique $D[[X]]$-homomorphism $\phi: D[[X]]\left[\left[Y_{l}\right]\right]$ $\rightarrow J[[X]]$ and we write $D[[X]][[\mathcal{F}]]$ to denote the image of $\phi$. We use $\omega$ to denote the set of positive integers. Thus, in (5) of (1.2) we have $D[[X]]\left[\left[\left\{Y_{i}\right\}_{i-1}^{\infty}\right]\right]=$ $D[[X]]\left[\left[Y_{\omega}\right]\right]$.

2. Main theorem. In $\$ 3$ we will give several conditions under which (1)-(5) of (1.2) are equivalent. In view of Proposition 3.7 it will suffice to show that (5) $\rightarrow$ (1) and our first result, Theorem 2.1, is the key result in this direction, for it gives necessary conditions in order that (5) of (1.2) hold for the domains $D \subset J$. For later convenience we actually state and prove Theorem 2.1 in the more general setting in which the domains $A \subset B$ satisfy (5) of (1.2), $A \subseteq D$ and $J=D[B]$. In the statement and proof of Theorem $2.1\left\{\gamma_{n}\right\}_{n=1}^{\infty}$ is a sequence of positive integers defined as follows. First let $\left\{\nu_{i j} \mid i \geqslant 1, j \geqslant i\right\}$ be a set of positive integers such that $\nu_{1,1}>1, \nu_{1, k}>k\left(1+\sum_{j<k, i \leqslant j} \nu_{i j}\right)$ for $k>1$, and $\nu_{i k}=\sum_{j=i-1}^{k} \nu_{i-1, j}$ for $i>1$. For each $k \geqslant 1$ we set $\gamma_{k}=\nu_{k k}$.

Theorem 2.1. Let $A$ and $B$ be integral domains such that $A \subset B$ and for each integral domain $R$ such that $A[[X]] \subset R \subseteq B[[X]]$ assume that $R \cong A[[X]]\left[\left[Y_{\omega}\right]\right]$ via an $A[[X]]$-isomorphism. Let $D$ and.J be integral domains with quotient fields $K$ and $L$, respectively, and suppose that $A \subseteq D \subseteq J$ and $J=D[B]$. If $K_{0}$ is the maximal separable extension of $K$ in $L$ then $L$ is algebraic over $K, L / K_{0}$ is purely inseparable with finite exponent, and $\left[K_{0}: K\right]$ is finite. Further, if $D_{0}$ is any integral domain with quotient field containing $K_{0}$ and such that $D \subseteq D_{0} \subseteq J$ then for each subset $\left\{\xi_{i}\right\}_{i=1}^{\infty}$ of 
$B$ there exist integers $n \geqslant 1$ and $m \geqslant 0$ and a nonzero element $d$ in $D$ such that for each $k \geqslant 1, d \xi_{k}^{p^{m}}$ is a polynomial in $D_{0}\left[\xi_{1}^{p^{m}}, \ldots, \xi_{k-1}^{p^{m}}\right]$ with total degree not more than $p^{m} \gamma_{k+n}$ in $\xi_{1}^{p^{m}}, \ldots, \xi_{k-1}^{p^{m}}$.

In proving Theorem 2.1 we let $\left\{\xi_{i}\right\}_{i=1}^{\infty}$ be a subset of $B$ and define a set $\mathscr{F}=\left\{f_{i}\right\}_{i=1}^{\infty} \subseteq A\left[\left\{\xi_{i}\right\}_{i=1}^{\infty}\right][[X]]$ such that $o\left(f_{i}\right) \geqslant i$. By assumption the homomorphism $\phi: A[[X]]\left[\left[Y_{\omega}\right]\right] \rightarrow A[[X]][[\mathcal{F}]]$ defined by $\phi\left(Y_{i}\right)=f_{i}$ is not an isomorphism. Therefore, there exists a nonzero power series $G(Y)$ in $A[[X]]\left[\left[Y_{\omega}\right]\right]$ such that $G(\mathscr{F})=0$. In $G(\mathscr{F})$ we wish to examine the coefficients of selected powers of $X$ and thus obtain polynomial equations in the $\xi_{i}$. In order to simplify the description of the desired coefficients we first reduce, via Lemma 2.2 and Corollary 2.3, to the case in which we have a single element $f \in A\left[\left\{\xi_{i}\right\}_{i=1}^{\infty}\right][[X]]$ and a nonzero power series $H(Y)$ in $A_{1}[[Y]]$, where $A_{1}$ is an appropriate extension of $A[[X]]$, such that $H(f)=0$.

In order that we may simultaneously consider the cases $p=1$ and $p>1$, in our next result, Lemma 2.2, we consider a situation somewhat more general than that described above. Namely, for each positive integer $i$, let $\mathscr{F}_{i}$ be a countable (perhaps finite) subset of $X B[[X]]$ indexed by the set $\Delta_{i}$. Set $\mathscr{F}=\cup_{i=1}^{\infty} \mathscr{F}_{i}, \Delta=\cup_{i=1}^{\infty} \Delta_{i}$ and for each positive integer $k$ let $\mathscr{F}_{(k)}=\cup_{i=1}^{k} \mathscr{F}_{i}, \mathscr{F}^{(k)}=\bigcup_{i=k+1}^{\infty} \mathscr{F}_{i}, \Delta_{(k)}=$ $\cup_{i=1}^{k} \Delta_{i}$, and $\Delta^{(k)}=\cup_{i=k+1}^{\infty} \Delta_{i}$. We further assume that $\{f \in \mathscr{F} \mid o(f)<k\}$ is finite for each positive integer $k$.

Lemma 2.2. Let $A_{1}$ be an integral domain such that $A[[X]] \subseteq A_{1} \subseteq B[[X]]$ and let $G(Y)=\sum_{\alpha \in \Lambda(\Delta)} a_{\alpha} Y^{\alpha}$ be a nonzero element of $A_{1}\left[\left[Y_{\Delta}\right]\right]$ such that $G(\mathscr{F})=0$. Then there exists an integer $t$ and a nonzero power series $H(Y)=\Sigma_{\beta \in \Lambda\left(\Delta_{t}\right)} b_{\beta} Y^{\beta}$ in $A_{1}\left[\left[\mathscr{F}^{(t)}\right]\right]\left[\left[Y_{\Delta_{t}}\right]\right]$ such that $H\left(\mathscr{F}_{t}\right)=0$. Further, if $p>1$ and $\pi_{\lambda}(\alpha)<p$ for each $\lambda \in \Delta$ and for each $\alpha \in \Lambda(\Delta)$ such that $a_{\alpha} \neq 0$, then $\pi_{\lambda}(\beta)<p$ for each $\lambda \in \Delta_{\text {l }}$ and for each $\beta \in \Lambda\left(\Delta_{t}\right)$ such that $b_{\beta} \neq 0$.

Proof. Choose $\alpha_{0}$ so that $o\left(a_{\alpha_{0}}\right)=\min \left\{o\left(a_{\alpha}\right) \mid \alpha \in \Lambda(\Delta)\right\}$. Since $G(\mathscr{F})=0$ we have $o\left(a_{0}\right)=o\left(-\sum_{\alpha \in \Lambda(\Delta) \backslash(0)} a_{\alpha} \mathscr{F}^{\alpha}\right)$. But $o(f) \geqslant 1$ for each $f \in \mathscr{F}$ so it follows that $\alpha_{0} \neq 0$. Let $s$ be a positive integer such that $\pi_{\lambda}\left(\alpha_{0}\right)=0$ for each $\lambda \in \Delta^{(s)}$ and write $G(Y)=\sum_{\gamma \in \Lambda\left(\Delta_{(s)}\right)} g_{\gamma} Y^{\gamma} \in A_{1}\left[\left[Y_{\left.\Delta^{(s)}\right]}\right]\left[\left[Y_{\Delta_{(s)}}\right]\right]\right.$. Let $\gamma_{0} \in \Lambda\left(\Delta_{(s)}\right)$ be such that $\pi_{\lambda}\left(\gamma_{0}\right)=$ $\pi_{\lambda}\left(\alpha_{0}\right)$ for each $\lambda \in \Delta_{(s)}$. If we write $g_{\gamma_{0}}=\Sigma_{\eta \in \Lambda\left(\Delta^{(s)}\right)} d_{\eta} Y^{\eta} \in A_{1}\left[\left[Y_{\Delta^{(s)}}\right]\right]$ then $d_{0}=a_{\alpha_{0}}$ and for $\eta \in \Lambda\left(\Delta^{(s)}\right)$ each nonzero $d_{\eta}$ is one of the coefficients $a_{\alpha}$ of $G(Y)$. If $g_{\gamma_{0}}\left(\mathscr{F}^{(s)}\right)=0$ then $d_{0}=-\sum_{\eta \in \Lambda\left(\Delta^{(s)}\right)(0)} d_{\eta}\left(\mathscr{F}^{(s)}\right)^{\eta}$ and since $o(f) \geqslant 1$ for $f \in \mathscr{F}^{(s)}$ this contradicts that $o\left(d_{0}\right) \leqslant o\left(d_{\eta}\right)$ for each nonzero $d_{\eta}$. Therefore $g_{\gamma_{0}}\left(\mathscr{F}^{(s)}\right) \neq 0$ so if we set $c_{\gamma}=g_{\gamma}\left(\mathscr{F}^{(s)}\right)$ for each $\gamma \in \Lambda\left(\Delta_{(s)}\right)$ then we obtain a nonzero power series $G_{1}(Y)=\sum_{\gamma \in \Lambda\left(\Delta_{(s)}\right)} c_{\gamma} Y^{\gamma} \in A_{1}\left[\left[\mathscr{F}^{(s)}\right]\right]\left[\left[Y_{\Delta_{(s)}}\right]\right]$ such that $G_{1}\left(\mathscr{F}_{(s)}\right)=0$. Clearly, if $p>1$ and $\pi_{\lambda}(\alpha)<p$ for each $\lambda \in \Delta$ and for each $\alpha \in \Lambda(\Delta)$ such that $a_{\alpha} \neq 0$, then $\pi_{\lambda}(\gamma)<p$ for each $\lambda \in \Delta_{(s)}$ and for each $\gamma \in \Lambda\left(\Delta_{(s)}\right)$ such that $c_{\gamma} \neq 0$.

The existence of $G_{1}$ allows us to choose a minimal subset $\left\{t_{1}, \ldots, t_{r}\right\}$ of $\{1, \ldots, s\}$ for which there exists a nonzero power series $H_{1}(Y)=$ $\sum_{\gamma \in \Lambda\left(\Delta_{t_{1}} \cup \cdots \cup \Delta_{t_{r}}\right)} c_{\gamma} Y^{\gamma}$ in $A_{1}\left[\left[\mathscr{F}^{(s)}\right]\right]\left[\left[Y_{\Delta_{1} \cup \cdots \cup \Delta_{t_{r}}}\right]\right]$ with $H_{1}\left(\mathscr{F}_{t_{1}}, \ldots, \mathscr{F}_{t_{r}}\right)=0$. Further, we assume $H_{1}(Y)$ is chosen so that if $p>1$ then $\pi_{\lambda}(\gamma)<p$ for each $\lambda \in \Delta_{t_{1}} \cup \cdots \cup \Delta_{t_{r}}$ and for each $\gamma \in \Lambda\left(\Delta_{t_{1}} \cup \cdots \cup \Delta_{t_{r}}\right)$ such that $c_{\gamma} \neq 0$. If we 


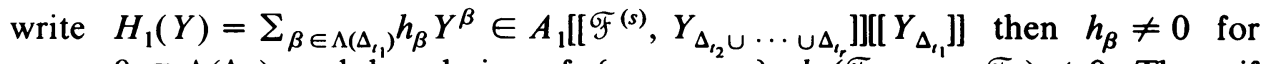
some $\beta \in \Lambda\left(\Delta_{t_{1}}\right)$ and by choice of $\left\{t_{1}, \ldots, t_{r}\right\}, h_{\beta}\left(\mathscr{F}_{t_{2}}, \ldots, \mathscr{F}_{t_{r}}\right) \neq 0$. Thus, if $b_{\beta}=h_{\beta}\left(\mathscr{F}_{t_{2}}, \ldots, \mathscr{F}_{t_{r}}\right)$ for each $\beta \in \Lambda\left(\Delta_{t_{1}}\right)$ then $H(Y)=\Sigma_{\beta \in \Lambda\left(\Delta_{t_{1}}\right)} b_{\beta} Y^{\beta} \in$ $A_{1}\left[\left[\mathscr{F}^{(s)}, \mathscr{F}_{t_{2}}, \ldots, \mathscr{F}_{t_{r}}\right]\right]\left[\left[Y_{\Delta_{t_{1}}}\right]\right] \subseteq A_{1}\left[\left[\mathscr{F}^{\left(t_{1}\right)}\right]\right]\left[\left[Y_{\Delta_{t_{1}}}\right]\right]$ is a nonzero power series such that $H\left(\mathscr{F}_{t_{1}}\right)=0$. Again it is clear that if $p>1$ then $\pi_{\lambda}(\beta)<p$ for each $\lambda \in \Delta_{t_{1}}$ and for each $\beta \in \Lambda\left(\Delta_{t_{1}}\right)$ such that $b_{\beta} \neq 0$.

Corollary 2.3. Let $\mathscr{F}=\left\{f_{i}\right\}_{i=1}^{\infty}$ be a subset of $X B[[X]]$ such that $o\left(f_{i}\right) \geqslant i$ for each $i$. If there exists a nonzero element $G(Y)$ in $A[[X]]\left[\left[Y_{\omega}\right]\right]$ such that $G(\mathscr{F})=0$ then there exist integers $s \geqslant 0$ and $t \geqslant 1$ and a nonzero element $H(Y)$ in $A\left[\left[X,\left\{f_{i}^{p^{j}} \mid j=s\right.\right.\right.$ and $i>t$ or $p>1$ and $\left.\left.\left.j>s\right\}\right]\right][[Y]]$ such that $H\left(f_{t}^{p^{s}}\right)=0$. Further, if $p>1$ then $H(Y)$ is a polynomial with degree less than $p$.

Proof. We first consider the case $p=1$. Thus, we wish to establish the existence of an integer $t$ and a nonzero element $H(Y)$ in $A\left[\left[X,\left\{f_{i}\right\}_{i=t+1}^{\infty}\right]\right][[Y]]$ such that $H\left(f_{t}\right)=0$. This is an immediate consequence of Lemma 2.2 with $\mathscr{F}_{i}=\left\{f_{i}\right\}$ for each $i \geqslant 1$.

Now assume that $p>1$ and suppose the notation is such that $0=G(\mathscr{F})=$ $\phi(G(Y))$ where $\phi: A[[X]]\left[\left[Y_{\omega}\right]\right] \rightarrow A[[X]][[\mathcal{F}]]$ is the homomorphism such that $\phi\left(Y_{i}\right)=f_{i}$ for each $i \in \omega$. For each positive integer $n$ we can, by the division algorithm, write $n$ uniquely in the form $n=n_{0}+n_{1} p+\cdots+n_{k} p^{k}$ where $0<n_{i}$ $<p$. Thus $Y_{i}^{n}=Y_{i}^{n_{0}}\left(Y_{i}^{p}\right)^{n_{1}} \cdots\left(Y_{i}^{p^{k}}\right)^{n_{k}}$ and we define a nonzero element $G_{1}(Y)$ in $A[[X]]\left[\left[Y_{\omega \times \omega}\right]\right]$ by replacing $Y_{i}^{n}$ in $G(Y)$ with $Y_{i, 1}^{n_{0}} \cdots Y_{i, k+1}^{n_{k}}$. For each positive integer $j$ set $\mathscr{F}_{j}=\left\{f_{i}^{p^{j-1}}\right\}_{i=1}^{\infty}$ and, changing the notation established in the corollary, let $\mathscr{F}=\cup_{j=1}^{\infty} \mathscr{F}_{j}$. If $\phi_{1}: A[[X]]\left[\left[Y_{\omega \times \omega}\right]\right] \rightarrow A[[X]][[\mathcal{F}]]$ is defined by $\phi_{1}\left(Y_{i j}\right)=f_{i}^{p^{j-1}}$ then $\phi(G)=\phi_{1}\left(G_{1}\right)=G_{1}(\mathscr{F})=0$. Further, if $G_{1}(Y)=$ $\sum_{\alpha \in \Lambda(\omega \times \omega)} a_{\alpha} Y_{\omega \times \omega}^{\alpha}$ then $\pi_{\lambda}(\alpha)<p$ for each $\lambda \in \omega . \times \omega$ and for each $\alpha \in \Lambda(\omega \times \omega)$ such that $a_{\alpha} \neq 0$. By Lemma 2.2 there exists an integer $s \geqslant 0$ and a nonzero power series $H_{1}(Y)=\sum_{\beta \in \Lambda(\omega)} b_{\beta} Y^{\beta}$ in $A\left[\left[X,\left\{f_{i}^{p^{j}}\right\}_{j=s+1}^{\infty}\right]\right]\left[\left[Y_{\omega}\right]\right]$ such that $H_{1}\left(\mathscr{F}_{s+1}\right)=0$ and such that $\pi_{\lambda}(\beta)<p$ for each $\lambda \in \omega$ and each $\beta \in \Lambda(\omega)$ such that $b_{\beta} \neq 0$. Set $A_{1}=A\left[\left[X,\left\{f_{i}^{p^{j}}\right\}_{j=s+1}^{\infty}\right]\right]$, for each positive integer $i$ let $\mathscr{F}_{i}^{\prime}=\left\{f_{i}^{p^{s}}\right\}$, and set $\mathscr{F}^{\prime}=$ $\bigcup_{i=1}^{\infty} \mathscr{F}_{i}^{\prime}=\left\{f_{i}^{p s}\right\}_{i=1}^{\infty}$. By Lemma 2.2 there exists a positive integer $t$ and a nonzero element $H(Y)=\sum_{i=0}^{\infty} c_{i} Y^{i}$ in $A_{1}\left[\left[\left\{f_{i}^{p^{s}}\right\}_{i=t+1}^{\infty}\right]\right][[Y]]$ such that $H\left(f_{t}^{p}\right)=0$ and $i<p$ if $c_{i} \neq 0$. This completes the proof of Corollary 2.3.

In our next two results, Lemma 2.5 and Corollary 2.7, we examine the coefficients of selected powers of $X$ in an expression of the form $H(f)=0$. Specifically, let $H(Y)=\sum_{i=0}^{\infty} a_{i}(X) Y^{i}$ be in $A_{1}[[Y]]$ where $A[[X]] \subseteq A_{1} \subseteq B[[X]]$ and where $a_{i}(X)=\sum_{j=0}^{\infty} y_{i j} X^{j}$ for each $i \geqslant 0$. Set $f=\sum_{i=t}^{\infty} \zeta_{i} X^{\nu_{i}}$ where $\left\{\zeta_{i}\right\}_{i=t}^{\infty}$ is a subset of $B$ and where $\left\{\nu_{i}\right\}_{i=t}^{\infty}$ is a sequence of positive integers such that $\nu_{t}>t \geqslant 1$ and $\nu_{k}>k\left(1+\sum_{i=t}^{k-1} \nu_{i}\right)$ for $k>t$. For a positive integer $n$ let $k_{1}<\cdots<k_{n}$ be a sequence of integers such that $k_{1}>t$ and for $1 \leqslant j \leqslant n$ set $\mu_{j}=\nu_{k_{1}}+\cdots+\nu_{k_{j}}$. In Lemma 2.5 we consider the coefficient of $X^{\mu_{n}}$ in $H(f)$.

If $g(X)=\sum_{i=0}^{\infty} b_{i} X^{\lambda_{i}}$ and if $\alpha$ is a nonnegative integer then $g_{(\alpha)}$ denotes the truncation $\sum_{i=0}^{\alpha} b_{i} X^{\lambda_{i}}$ of $g$. The following observation is easily proved by induction on $k$ (cf. $[1,3.2])$ and will be useful in the proof of Lemma 2.5. 
(2.4) If $\alpha$ and $\beta$ are integers with $t \leqslant \alpha<\beta$ then for each positive integer $k$ we can write

$$
f_{(\beta)}^{k}=f_{(\alpha)}^{k}+g+k \zeta_{\beta} X^{v_{\beta}} f_{(\alpha)}^{k-1}+h
$$

where $o(h) \geqslant \nu_{\beta}+\nu_{\alpha+1}, g=0$ if $\alpha=\beta-1, o(g) \geqslant \nu_{\alpha+1}$ if $\alpha<\beta-1$, and $g \in$ $D\left[\zeta_{t}, \ldots, \zeta_{\beta-1}\right][X]$.

We note that Lemma 2.5 is a slightly altered version of Lemma 3.1 of [1] and our proof parallels that given in [1].

LEMMA 2.5. With notation as above let $\left\{k_{i}\right\}_{i=1}^{n}$ be a fixed set of positive integers such that $t<k_{1}<\cdots<k_{n}$ and set $\mu_{n}=\nu_{k_{1}}+\cdots+\nu_{k_{n}}$. If $\phi$ is the coefficient of $X^{\mu_{n}}$ in $H(f)$ then $\phi$ determines polynomials $\left\{\phi_{i}\right\}_{i=0}^{n}$ and $\left\{\psi_{i}\right\}_{i=0}^{n-1}$ such that $\phi=\phi_{n}$ and the following conditions hold:

(1) For $0 \leqslant m<n, \phi_{m+1}=\phi_{m} \zeta_{k_{m+1}}+\psi_{m}$.

(2) For $0 \leqslant m<n$, $\phi_{m}$ is a polynomial in $A\left[\left\{y_{i j} \mid 0 \leqslant i \leqslant \mu_{n}, 0 \leqslant j \leqslant \mu_{m}\right\}\right.$, $\left.\zeta_{t}, \ldots, \zeta_{k_{m}}\right]$ and is independent of the choice of $k_{m+1}, \ldots, k_{n}$.

(3) For $0 \leqslant m<n, \psi_{m}$ is a polynomial in $A\left[\left\{y_{i j} \mid 0 \leqslant i \leqslant \mu_{n}, 0 \leqslant j \leqslant \mu_{m+1}\right\}\right.$, $\left.\zeta_{t}, \ldots, \zeta_{k_{m+1}-1}\right]$ which has total degree one in the $y_{i j}$ and total degree not greater than $\mu_{n}$ in the $\zeta_{i}$.

(4) $\phi_{0}=n ! y_{n, 0}$.

Proof. Let $\phi_{s n}$ denote the coefficient of $X^{\mu_{n}}$ in $a_{s} f^{s}$. If $s>\mu_{n}$ then $o\left(f^{s}\right)>\mu_{n}$ so $\phi_{s n}=0$. Thus, we assume that $0 \leqslant s \leqslant \mu_{n}$. Clearly the coefficient of $X^{\mu_{n}}$ in $H(f)$ is $\phi_{n}=\sum_{s=0}^{\mu_{n}} \phi_{s n}$ so the lemma follows if we prove that each $\phi_{s n}$ determines polynomials $\left\{\phi_{s i}\right\}_{i=0}^{n}$ and $\left\{\psi_{s i}\right\}_{i=0}^{n-1}$ that satisfy conditions (1)-(3) and have the property that $\phi_{s, 0}=0$ if $s \neq n$ while $\phi_{n, 0}=n ! y_{n, 0}$. For the remainder of the proof we are, therefore, interested only in the coefficient of $X^{\mu_{n}}$ in $a_{s} f^{s}$. Thus, we fix $s$ and drop it from the notation $\phi_{s i}$ and $\psi_{s i}$. The following result will simplify the process of describing the polynomials $\left\{\phi_{i}\right\}_{i=0}^{n}$ and $\left\{\psi_{i}\right\}_{i=0}^{n-1}$.

(2.6) For $s<n$ and $n-s \leqslant m \leqslant n$ and for $s \geqslant n$ and $1 \leqslant m \leqslant n$ set $P_{m}(X)=$ $\left(a_{s}\right)_{\left(\mu_{m}\right)}\left[(s ! /(s-n+m) !) f_{\left(k_{m}\right)}^{s-n+m}\right]$. If $m<n$ then $P_{m+1}(X)=Q_{m}(X)+$ $P_{m}(X) \zeta_{k_{m+1}} X^{\nu_{k_{m+1}}}+H_{m}(X)$ where $o\left(H_{m}\right)>\mu_{m+1}$ and $Q_{m}(X)$ has coefficients in $A\left[\left\{y_{s j}\right\}_{j=0}^{\mu_{m+1}}, \zeta_{t}, \ldots, \zeta_{k_{m+1}-1}\right]$ with total degree one in the $y_{s j}$ and total degree not greater than $\mu_{n}$ in the $\zeta_{i}$.

To prove (2.6) suppose that $m<n$. By assumption we have $P_{m+1}(X)=$ $\left(a_{s}\right)_{\left(\mu_{m+1}\right)}\left[(s ! /(s-n+m+1) !) f_{\left(k_{m+1}\right)}^{s-n+m+1}\right]$ and by (2.4) we can write $f_{\left(k_{m+1}\right)}^{s-n+m+1}=$

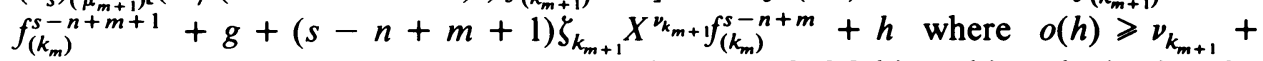
$\nu_{k_{m}+1}, o(g) \geqslant \nu_{k_{m}+1}$, and $g \in D\left[\zeta_{t}, \ldots, \zeta_{k_{m+1}-1}\right][X]$. Making this substitution for $f_{\left(k_{m+1}\right)}^{s-n+1}$ in $P_{m+1}(X)$ we may write $P_{m+1}(X)=Q_{m}(X)+P_{m}(X) \zeta_{k_{m+1}} X^{v_{k_{m+1}}}+$ $H_{m}(X)$ where $Q_{m}(X)=\left(a_{s}\right)_{\left(\mu_{m+1}\right)}\left[(s ! /(s-n+m+1) !)\left(f_{\left(k_{m}\right)}^{s-n+m+1}+g\right)\right]$ and

$$
\begin{aligned}
H_{m}(X)= & \left(\sum_{j=\mu_{m}+1}^{\mu_{m+1}} y_{s j} X^{j}\right)\left[(s ! /(s-n+m) !) \zeta_{k_{m+1}} X^{\left.\nu_{k_{m}+1} f_{\left(k_{m}\right)}^{s-n+m}\right]}\right. \\
& +\left(a_{s}\right)_{\left(\mu_{m+1}\right)}[(s ! /(s-n+m+1) !) h] .
\end{aligned}
$$

Now $o(h) \geqslant \nu_{k_{m+1}}+\nu_{k_{m}+1}>\nu_{k_{m+1}}+\sum_{i=t}^{k_{m}} \nu_{i} \geqslant \nu_{k_{1}}+\cdots+\nu_{k_{m+1}}=\mu_{m+1}$ and $\mu_{m}+$ $1+\nu_{k_{m+1}}>\mu_{m}+\nu_{k_{m+1}}=\mu_{m+1}$, so $o\left(H_{m}\right)>\mu_{m+1}$. Our hypothesis on $g$ implies that 
$Q_{m}(X)$ has coefficients in $A\left[\left\{y_{s j}\right\}_{j=0}^{\mu_{m+1}}, \zeta_{t}, \ldots, \zeta_{k_{m+1}-1}\right]$ with total degree one in the $y_{s j}$ and total degree $s-n+m+1$ in the $\zeta_{i}$. But $s-n+m+1 \leqslant s \leqslant \mu_{n}$ and the proof of (2.6) is complete.

By assumption $\nu_{k_{n}+1}>\left(k_{n}+1\right)\left[1+\sum_{i=t}^{k_{n}} \nu_{i}\right] \geqslant \mu_{n}$ so in determining the coefficient of $X^{\mu_{n}}$ in $a_{s} f_{s}$ it suffices to consider $\left(a_{s}\right)_{\left(\mu_{n}\right)} f_{\left(k_{n}\right)}^{s}=P_{n}(X)$; that is, $\phi_{n}$ is the coefficient of $X^{\mu_{n}}$ in $P_{n}(X)$. If $s<n$ and $n-s \leqslant m<n$ or if $s>n$ and $1 \leqslant m<n$ then let $\phi_{m}$ denote the coefficient of $X^{\mu_{m}}$ in $P_{m}(X)$. It is an immediate consequence of (2.6) that $\phi_{m+1}=\phi_{m} \zeta_{k_{m+1}}+\psi_{m}$ where $\phi_{m} \zeta_{k_{m+1}}$ is the coefficient of $X^{\mu_{m+1}}=$ $X^{\mu_{m}+v_{k_{m+1}}}$ in $P_{m}(X) \zeta_{k_{m+1}} X^{\nu_{k_{m+1}}}$ and $\psi_{m}$ is the coefficient of $X^{\mu_{m+1}}$ in $Q_{m}(X)$. Thus, (1) of Lemma 2.5 is satisfied by the polynomials $\phi_{m+1}, \phi_{m}$, and $\psi_{m}$. If $m<n$ then $P_{m}(X)$ is clearly independent of the choice of $k_{m+1}, \ldots, k_{n}$ and, hence, so is $\phi_{m}$. It is straightforward to see that $\phi_{m} \in D\left[\left\{y_{s j}\right\}_{j=0}^{\mu_{m}}, \zeta_{t}, \ldots, \zeta_{k_{m}}\right]$ so (2) of Lemma 2.5 holds. That (3) holds for the polynomials $\psi_{m}$ is immediate from (2.6).

If $s<n$ we have determined polynomials $\left\{\phi_{i}\right\}_{i=n-s}^{n}$ and $\left\{\psi_{i}\right\}_{i=n-s}^{n-1}$ while for $s \geqslant n$ we have determined polynomials $\left\{\phi_{i}\right\}_{i=1}^{n}$ and $\left\{\psi_{i}\right\}_{i=1}^{n-1}$ that satisfy (1)-(3) of Lemma 2.5. If $s<n$ and $m=n-s$ then, by assumption, $\phi_{m}$ is the coefficient of $X^{\mu_{m}}$ in $P_{m}(X)=s !\left(a_{s}\right)_{\left(\mu_{m}\right)}$. Thus, we have $\phi_{n-s}=s ! y_{s \mu_{m}}$. We take $\psi_{n-s-1}=\phi_{n-s}$, $\phi_{i}=0$ for $0 \leqslant i<n-s$ and $\psi_{i}=0$ for $0 \leqslant i<n-s-1$. If $s \geqslant n$ then, by assumption, $\phi_{1}$ is the coefficient of $X^{\mu_{1}}$ in

$$
P_{1}(X)=\left(a_{s}\right)_{\left(\mu_{1}\right)}\left[(s ! /(s-n+1) !) f_{\left(k_{1}\right)}^{s-n+1}\right] .
$$

By (2.4) we can write $f_{\left(k_{1}\right)}^{s-n+1}=f_{\left(k_{1}-1\right)}^{s-n+1}+(s-n+1) \zeta_{k_{1}} X^{v_{k_{1}}} f_{\left(k_{1}-1\right)}^{s-n}+h$ where $o(h)$ $\geqslant 2 v_{k_{1}}>\mu_{1}$. If $s>n$ then $o\left(X^{v_{k_{1}}} f_{\left(k_{1}-1\right)}^{s-n}\right) \geqslant \nu_{k_{1}}+1>\nu_{k_{1}}=\mu_{1}$ so $\phi_{1}$ is the coefficient of $X^{\mu_{1}}$ in $\left(a_{s}\right)_{\left(\mu_{1}\right)}\left[(s ! /(s-n+1) !) f_{\left(k_{1}-1\right)}^{s-n+1}\right]$. Thus, we set $\psi_{0}=\phi_{1}$ and $\phi_{0}=0$ and it is straightforward to see that (3) of Lemma 2.5 holds for $\psi_{0}$. Finally, if $s=n$ then $\phi_{1}$ is the coefficient of $X^{\mu_{1}}$ in $P_{1}(X)=\left(a_{n}\right)_{\left(\mu_{1}\right)} n ! f_{\left(k_{1}\right)}=$ $\left(a_{n}\right)_{\left(\mu_{1}\right)} n !\left[f_{\left(k_{1}-1\right)}+\zeta_{k_{1}} X^{\left.\nu_{k_{1}}\right]}\right.$ so we can write $\phi_{1}=n ! y_{n, 0} \zeta_{k_{1}}+\psi_{0}$ where $\psi_{0}$ is the coefficient of $X^{\mu_{1}}$ in $\left(a_{n}\right)_{\left(\mu_{1}\right)} n ! f_{\left(k_{1}-1\right)}$. In particular, $\psi_{0} \in A\left[\left\{y_{n j}\right\}_{j=0}^{\mu_{1}}, \zeta_{t}, \ldots, \zeta_{k_{1}-1}\right]$ with total degree one in the $y_{n j}$ and total degree not greater than $\mu_{n}$ in the $\zeta_{i}$. We set $\phi_{0}=n ! y_{n, 0}$ and the proof of Lemma 2.5 is complete.

Let $\left\{\zeta_{i}\right\}_{i=1}^{\infty}$ be a subset of $B$ and for each positive integer $n$ set $\mathcal{E}_{n}=\{g(X)=$ $\sum_{i=t}^{\infty} \alpha_{i} X^{\lambda_{i}} \mid o(g) \geqslant n$ and for each $i \geqslant t, \alpha_{i} \in A\left[\zeta_{1}, \ldots, \zeta_{i}\right]$ with total degree $n$ in $\left.\zeta_{1}, \ldots, \zeta_{i}\right\}$. If $g=\sum_{i=t}^{\infty} \alpha_{i} X^{\lambda_{i}} \in \mathcal{E}_{n_{1}}$ and $h=\sum_{i=s}^{\infty} \beta_{i} X^{\gamma_{i}} \in \mathcal{E}_{n_{2}}$ we say that $h$ dominates $g$ provided $n_{1} \leqslant n_{2}$ and $\gamma_{i} \geqslant \sum_{j=t}^{i} \lambda_{j}$ for $i \geqslant \max \{s, t\}$. Set $\mathscr{E}=$ $\cup_{n=1}^{\infty} \varepsilon_{n}$.

Corollary 2.7. Let $f(X)=\sum_{i=t}^{\infty} \zeta_{i} X^{v_{i}}$ where $\nu_{t}>t \geqslant 1$ and $\nu_{k}>k\left(1+\sum_{i=t}^{k-1} \nu_{i}\right)$ for $k>t$. Assume that $G(Y)=\sum_{i=0}^{\infty} b_{i} Y^{i}$ is a nonzero element of $A\left[\left[X,\left\{q_{\lambda}\right\}_{\lambda \in \Gamma}\right]\right][[Y]]$, where $\left\{q_{\lambda}\right\}_{\lambda \in \Gamma}$ is a countable subset of $\mathcal{E}$ such that each $q_{\lambda}$ dominates $f$ and such that $\left\{q_{\lambda} \mid o\left(q_{\lambda}\right) \leqslant k\right\}$ is finite for each positive integer $k$. Choose $n$ such that $o\left(b_{n}\right)=\min \left\{o\left(b_{i}\right)\right\}_{i=1}^{\infty}$ and if $A$ has nonzero characteristic $p$ assume that we can choose $n<p$. If $G(f)=0$ then there exists a positive integer $k_{0}>t$ and a nonzero element $d$ in $A\left[\zeta_{1}, \ldots, \zeta_{k_{0}}\right]$ such that for $k>k_{0}, d \zeta_{k}$ is a polynomial in $A\left[\zeta_{1}, \ldots, \zeta_{k-1}\right]$ with total degree not more than $\nu_{k+n}$ in $\zeta_{1}, \ldots, \zeta_{k-1}$. 
Proof. Let $N=o\left(b_{n}\right)=\min \left\{o\left(b_{i}\right)\right\}_{i=1}^{\infty}$ where, by assumption, $n<p$ if $p>1$. Since $b_{0}=-\sum_{i=1}^{\infty} b_{i} f^{i}$ and $o(f) \geqslant 1$ we have $o\left(b_{0}\right)>N$. Thus, for each $i \geqslant 0$ we can write $b_{i}=\sum_{j=0}^{\infty} y_{i j} X^{j+N}$ where $y_{i j} \in B$ and $y_{n, 0} \neq 0$. If we set $a_{i}=b_{i} / X^{N}=$ $\sum_{j=0}^{\infty} y_{i j} X^{j}$ and $H(Y)=G(Y) / X^{N}$, then $H(Y)=\sum_{i=0}^{\infty} a_{i} Y^{i}$ and $H(f)=0$. We apply Lemma 2.5 to $H(f)$.

Let $\delta$ denote the set of all sequences $\left\{k_{i}\right\}_{i=1}^{n}$ (where $n$ is as chosen above) of positive integers such that $\max \{N, t\}<k_{1}<\cdots<k_{n}$. For $\sigma \in \mathcal{S}$ we denote by $\left\{{ }_{\sigma} \phi_{i}\right\}_{i=0}^{n}$ and $\left\{{ }_{\sigma} \psi_{i}\right\}_{i=0}^{n-1}$ the collection of polynomials obtained as in Lemma 2.5 by considering the coefficient of $X^{\mu_{n}}$ in $H(f)$, where $\mu_{n}=\nu_{k_{1}}+\cdots+\nu_{k_{n}}$. This coefficient is necessarily zero so for each $\sigma \in \mathcal{S}$ we have ${ }_{\sigma} \phi_{n}=0$. Also, for each $\sigma \in \mathcal{S},{ }_{\sigma} \phi_{0}=n ! y_{n, 0}$ by (4) of Lemma 2.5. Since $n<p$ if $p>1,{ }_{\sigma} \phi_{0} \neq 0$. Therefore there exists a largest integer $m, 0 \leqslant m<n$, for which there is a sequence $\tau=$ $\left\{k_{i}^{0}\right\}_{i=1}^{n}$ in $\delta$ with ${ }_{\tau} \phi_{m} \neq 0$. By (2) of Lemma 2.5, ${ }_{\tau} \phi_{m}$ is independent of the choice of $k_{m+1}^{0}, \ldots, k_{n}^{0}$; that is, if $\sigma=\left\{k_{1}^{0}, \ldots, k_{m}^{0}, k_{m+1}, \ldots, k_{n}\right\} \in \mathcal{S}$ then ${ }_{\sigma} \phi_{m}={ }_{\tau} \phi_{m}$ $\neq 0$. Moreover, by choice of $m,{ }_{\sigma} \phi_{i}=0$ for $m+1 \leqslant i \leqslant n$. If $k$ is an integer such that $k>k_{m}^{0}$ then we may choose $\sigma=\left\{k_{i}\right\}_{i=1}^{n}$ in $\mathcal{S}$ such that $k_{i}=k_{i}^{0}$ for $1 \leqslant i \leqslant m$ and $k_{m+i}=k+i-1$ for $1 \leqslant i \leqslant n-m$. For $1 \leqslant j \leqslant n$ set $\mu_{j}=\nu_{k_{1}}+\cdots+\nu_{k_{j}}$. By Lemma 2.5 we have

$$
\begin{aligned}
& 0={ }_{\sigma} \phi_{m+1}={ }_{\sigma} \phi_{m} \zeta_{k_{m+1}}+{ }_{\sigma} \psi_{m}={ }_{\sigma} \phi_{m} \zeta_{k}+{ }_{\sigma} \psi_{m},{ }_{\sigma} \phi_{m} \\
& \quad \in A\left[\left\{y_{i j} \mid 0 \leqslant i \leqslant \mu_{n}, 0 \leqslant j \leqslant \mu_{m}\right\}, \zeta_{t}, \ldots, \zeta_{k_{m}}\right]
\end{aligned}
$$

and

$$
{ }_{\sigma} \psi_{m} \in A\left[\left\{y_{i j} \mid 0 \leqslant i \leqslant \mu_{n}, 0 \leqslant j \leqslant \mu_{m+1}\right\}, \zeta_{t}, \ldots, \zeta_{k-1}\right]
$$

with total degree one in the $y_{i j}$ and total degree not greater than $\mu_{n}$ in the $\zeta_{i}$.

For each $i \geqslant 0, b_{i} \in A\left[\left[X,\left\{q_{\lambda}\right\}_{\lambda \in \Gamma}\right]\right]$ so we can write $b_{i}=\sum_{j=0}^{\infty} y_{i j} X^{j+N}=$ $h_{i}\left(X,\left\{q_{\lambda}\right\}_{\lambda \in \Gamma}\right)$. For $1 \leqslant s \leqslant n$ and $0 \leqslant j \leqslant \mu_{s}$ we wish to further describe $y_{i j}$. Suppose that $q_{\lambda}=\sum_{i=t_{\lambda}}^{\infty} \beta_{\lambda i} X^{\gamma_{\lambda, i}}$ for each $\lambda \in \Gamma$. If $i \geqslant \max \left\{t_{\lambda}, k_{s}\right\}$ then, since $q_{\lambda}$ dominates $f$, we have $\gamma_{\lambda i} \geqslant \sum_{j=t}^{i} \nu_{j} \geqslant \sum_{j=t}^{k_{s}} \nu_{i} \geqslant \nu_{k_{1}-1}+\nu_{k_{1}}+\cdots+\nu_{k_{s}}=\nu_{k_{1}-1}+$ $\mu_{s}>\left(k_{1}-1\right)\left(1+\sum_{j=t}^{k_{1}-2} \nu_{j}\right)+\mu_{s} \geqslant N+\mu_{s}$ since by choice $k_{1}>N$. Therefore, in computing the coefficients $y_{i j}$ of $X^{j+N}$ for $0 \leqslant j \leqslant \mu_{s}$, we can replace $q_{\lambda}$ with $\left(q_{\lambda}\right)_{\left(\gamma_{\left.\lambda, k_{s-1}\right)}\right)}=\sum_{i=t_{\lambda}}^{k_{s-1}} \beta_{\lambda i} X^{\gamma_{\lambda, i}}$. But $q_{\lambda} \in \mathcal{E}$ so for $i \geqslant t_{\lambda}$ we have $\beta_{\lambda i} \in A\left[\zeta_{1}, \ldots, \zeta_{i}\right]$; therefore, $y_{i j} \in A\left[\zeta_{1}, \ldots, \zeta_{k_{s}-1}\right]$ for $0 \leqslant j \leqslant \mu_{s}$. But this implies that ${ }_{\sigma} \phi_{m} \in$ $A\left[\zeta_{1}, \ldots, \zeta_{k_{m}}\right]$ and ${ }_{\sigma} \psi_{m} \in A\left[\zeta_{1}, \ldots, \zeta_{k-1}\right]$. For $0 \leqslant j \leqslant \mu_{s}$ we now determine an upper bound on the degree of $y_{i j}$ as a polynomial in the $\zeta_{i}$. In $b_{i}=\sum_{j=0}^{\infty} y_{i j} X^{j+N}=$ $h_{i}\left(X,\left\{q_{\lambda}\right\}_{\lambda \in \Gamma}\right)$ suppose we consider a monomial $q_{\lambda_{1}}^{m_{1}} \cdots q_{\lambda_{r}}^{m_{r}}$. If $q_{\lambda_{i}} \in \mathcal{E}_{n_{i}}$ then $o\left(q_{\lambda_{i}}\right) \geqslant n_{i}$ so $o\left(q_{\lambda_{1}}^{m_{1}} \cdots q_{\lambda_{r}}^{m_{r}}\right) \geqslant \sum_{i=1}^{r} n_{i} m_{i}$. Clearly then, only those monomials such that $\sum_{i=1}^{r} n_{i} m_{i} \leqslant \mu_{s}+N$ can contribute to $y_{i j}$ for $0 \leqslant j \leqslant \mu_{s}$. Since the coefficients of $q_{\lambda_{i}}$ have total degree $n_{i}$ in the $\zeta_{j}$, the coefficients of the monomial $q_{\lambda_{1}}^{m_{1}} \cdots q_{\lambda_{r}}^{m_{r}}$ have total degree $\sum_{i=1}^{r} n_{i} m_{i} \leqslant \mu_{s}+N$ in the $\zeta_{j}$. Consequently, $y_{i j}$ has total degree at most $\mu_{s}+N$ in $\zeta_{1}, \ldots, \zeta_{k_{s}-1}$ so ${ }_{\sigma} \psi_{m}$ has total degree at most $\mu_{n}+\mu_{m+1}+N \leqslant 2 \mu_{n}+N$ in $\zeta_{1}, \ldots, \zeta_{k-1}$. Since $k_{n}=k+n-m-1<k+n$ we have $\nu_{k+n}>(k+n)\left(1+\sum_{i=t}^{k+n-1} \nu_{i}\right) \geqslant N+2 \sum_{i=t}^{k+n-1} \nu_{i} \geqslant N+2 \mu_{n}$. If we take $d={ }_{\sigma} \phi_{m}$ and $k_{0}=k_{m}$ the proof of Corollary 2.7 is complete. 
Let $\left\{\xi_{i}\right\}_{i=1}^{\infty}$ be a subset of $B$, let $\left\{v_{i j} \mid i \geqslant 1, j \geqslant i\right\}$ and $\left\{\gamma_{i}\right\}_{i=1}^{\infty}$ denote the sets of positive integers as described preceding the statement of Theorem 2.1, and let $\mathscr{F}=\left\{f_{i}\right\}_{i=1}^{\infty}$ be the subset of $B[[X]]$ defined by $f_{i}=\sum_{j-i}^{\infty} \xi_{j} X^{\nu_{i j}}$. By assumption there exists a nonzero power series $G(Y)$ in $A[[X]]\left[\left[Y_{\omega}\right]\right]$ such that $G(\mathscr{F})=0$. By Corollary 2.3 there exist integers $m \geqslant 0$ and $t \geqslant 1$ and a nonzero element $H(Y)$ in $A\left[\left[X,\left\{f_{i}^{p^{j}} \mid j=m\right.\right.\right.$ and $i>t$ or $p>1$ and $\left.\left.\left.\left.j>m\right\}\right]\right][Y]\right]$ such that $H\left(f_{t}^{p^{m}}\right)=0$. Further, if $p>1$ then $H(Y)$ is a polynomial with degree less than $p$. Define $f=f_{t}^{p^{m}}=\sum_{j=t}^{\infty} \zeta_{j} X^{\nu_{j}}$ where $\zeta_{j}=\xi_{j}^{p^{m}}$ and $\nu_{j}=p^{m} \nu_{t j}$. Then $\nu_{t}=p^{m} \nu_{t t}>t$ and for $k>t, \nu_{k}=p^{m} \nu_{t k} \geqslant p^{m} \nu_{1 k}>p^{m} k\left(1+\sum_{j=t}^{k-1} \nu_{t j}\right) \geqslant k\left(1+\sum_{j=t}^{k-1} \nu_{j}\right)$. If $j>m$, with $j$

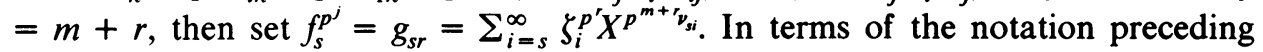
Corollary 2.7 we have $g_{s r} \in \mathcal{E}_{p^{r}}$. If $r=0$ then $j=m$ and $s>t$ so $p^{m} v_{s i}=$ $p^{m} \sum_{\lambda=s-1}^{i} \nu_{s-1, \lambda} \geqslant \sum_{\lambda=t}^{i} p^{m} \nu_{t \lambda}=\sum_{\lambda=t}^{i} \nu_{\lambda}$ and hence $g_{s, 0}$ dominates $f$ for $s>t$. If $r>0$ then $j>m$ and $p>1$ so $p^{r} \nu_{s i} \geqslant 2 \nu_{1, i}>\nu_{1, i}+i\left(1+\Sigma_{\lambda<i, \delta<\lambda} \nu_{\delta \lambda}\right) \geqslant \nu_{1, i}+$ $\sum_{\delta=1}^{i-1} \sum_{\lambda=\delta}^{i-1} \nu_{\delta \lambda}$. But if $\delta>1$ then $\nu_{\delta i}=\sum_{\lambda=\delta-1}^{i} \nu_{\delta-1, i}$ so if $1 \leqslant \rho \leqslant i$ the last expression of the previous sentence equals $\nu_{\rho i}+\sum_{\delta=\rho}^{i-1} \sum_{\lambda=\delta}^{i-1} \nu_{\delta \lambda} \geqslant \sum_{\lambda=\rho}^{i} \nu_{\rho \lambda}$. In particular, if $i \geqslant t$ then, with $\rho=t$, we get that $p^{m+r} \nu_{s i}>p^{m} \sum_{\lambda-t}^{i} \nu_{t \lambda}=\sum_{\lambda-t}^{i} \nu_{\lambda}$. Hence, $g_{s r}$ dominates $f$ when $r>0$. By Corollary 2.7 there exist integers $n$ and $k_{0}$ and a nonzero element $d$ in $A\left[\zeta_{1}, \ldots, \zeta_{k_{0}}\right]$ such that for $k>k_{0}, d \zeta_{k}$ is a polynomial in $A\left[\zeta_{1}, \ldots, \zeta_{k-1}\right]$ with total degree not more than $v_{k+n}$ in $\zeta_{1}, \ldots, \zeta_{k-1}$. But $\nu_{k+n}=p^{m} \nu_{t, k+n} \leqslant p^{m} \nu_{k+n, k+n}=p^{m} \gamma_{k+n}$ and $\zeta_{i}=\xi_{i}^{p^{m}}$ so we have proved the following.

(2.8) For each subset $\left\{\xi_{i}\right\}_{i=1}^{\infty}$ of $B$ there exist integers $n \geqslant 1, k_{0} \geqslant 1$, and $m \geqslant 0$ and a nonzero element $d$ in $A\left[\xi_{1}^{p^{m}}, \ldots, \xi_{k_{0}}^{p^{m}}\right]$ such that for $k>k_{0}, d \xi_{k}^{p^{m}}$ is a polynomial in $A\left[\xi_{1}^{p^{m}}, \ldots, \xi_{k-1}^{p^{m}}\right]$ with total degree not more than $p^{m} \gamma_{k+n}$ in $\xi_{1}^{p^{m}}, \ldots, \xi_{k-1}^{p^{m}}$.

Recall that $A \subseteq D \subseteq J, J=D[B], D$ and $J$ have quotient fields $K$ and $L$, respectively, and $K_{0}$ is the maximal, separable extension of $K$ in $L$.

(2.9) $L$ is algebraic over $K, L / K_{0}$ is purely inseparable with finite exponent, and $\left[K_{0}: K\right]$ is finite.

Proof. To show that $L / K$ is algebraic, it suffices to show that $J$ is algebraic over $D$. But since $J=D[B]$ and $A \subseteq D$, it suffices to show that $B$ is algebraic over $A$. Thus, let $\zeta \in B$ and, with $\left\{\gamma_{i}\right\}_{i=1}^{\infty}$ as defined in the remarks preceding the statement of Theorem 2.1, let $\xi_{k}=\zeta^{\gamma_{2 k}^{k}}$ for each integer $k \geqslant 1$. By (2.8) there exist integers $n \geqslant 1, k_{0} \geqslant 1, m \geqslant 0$ and a nonzero element $d$ in $A\left[\xi_{1}^{p^{m}}, \ldots, \xi_{k_{0}}^{p^{m}}\right]$ such that, for $k>k_{0}, d \xi_{k}^{p^{m}}$ is a polynomial in $A\left[\xi_{1}^{p^{m}}, \ldots, \xi_{k-1}^{p^{m}}\right]$ with total degree not more than $p^{m} \gamma_{k+n}$ in $\xi_{1}^{p^{m}}, \ldots, \xi_{k-1}^{p^{m}}$. Since $\xi_{k-1}^{p^{m}}=\zeta^{p^{m} \gamma_{2 k-1}^{k}}$ it follows that $d \xi_{k}^{p^{m}}=$ $d \zeta^{p^{m} \gamma_{2 k}^{k}}$ has total degree at most $p^{2 m} \gamma_{k+n} \gamma_{2 k-2}^{k-1}$ in $\zeta$ and if we choose $k>$ $\max \left\{n, p^{m}\right\}$ then $\gamma_{2 k}=\nu_{2 k, 2 k} \geqslant \nu_{1,2 k}>k v_{k+n, k+n}=k \gamma_{k+n}>p^{m} \gamma_{k+n}$. Thus, $p^{2 m} \gamma_{k+n} \gamma_{2 k-2}^{k-1}<p^{m} \gamma_{2 k} \gamma_{2 k}^{k-1}=p^{m} \gamma_{2 k}^{k}$. Since $d$ is a nonzero polynomial in $\zeta$ it follows that $\zeta$ is algebraic over $A$.

We now show the existence of a finite extension $K_{1}$ of $K$ such that $K_{1} \subseteq L$ and $L / K_{1}$ is purely inseparable with finite exponent. If $m \geqslant 0$ is an integer then $L^{p^{m}} \subseteq K_{1}$ if and only if $J^{p^{m}} \subseteq K_{1}$ and since $J=D[B], J^{p^{m}} \subseteq K_{1}$ if and only if $B^{p^{m}} \subseteq K_{1}$. Thus, if the desired extension $K_{1}$ does not exist we can choose a subset 
$\left\{\xi_{i}\right\}_{i=1}^{\infty}$ of $B$ such that $\xi_{k}^{p^{k}}$ is not in the quotient field of $D\left[\xi_{1}, \ldots, \xi_{k-1}\right]$. But by (2.8) there exist integers $n \geqslant 1, k_{0} \geqslant 1$, and $m \geqslant 0$ and a nonzero element $d$ in $A\left[\xi_{1}^{p^{m}}, \ldots, \xi_{k_{0}}^{p^{m}}\right]$ such that, for $k>k_{0}, d \xi_{k}^{p^{m}}$ is a polynomial in $A\left[\xi_{1}^{p^{m}}, \ldots, \xi_{k-1}^{p^{m}}\right] \subseteq$ $D\left[\xi_{1}, \ldots, \xi_{k-1}\right]$. In particular, $\xi_{k}^{p^{m}}$ is in the quotient field of $D\left[\xi_{1}, \ldots, \xi_{k-1}\right]$ for $k \geqslant \max \left\{m, k_{0}\right\}$ contrary to the choice of the set $\left\{\xi_{i}\right\}_{i=1}^{\infty}$, so we conclude that the desired extension $K_{1}$ of $K$ exists. Let $K_{0}$ be the maximal separable extension of $K$ in $K_{1}$. Then $\left[K_{0}: K\right]$ is finite and since $\left[K_{1}: K_{0}\right]$ is finite, $K_{1} / K_{0}$ has finite exponent. It follows that $L / K_{0}$ is purely inseparable with finite exponent and hence $K_{0}$ is the maximal separable extension of $K$ in $L$. This completes the proof of (2.9).

Now let $D_{0}$ be any integral domain with quotient field $K_{1}$ containing $K_{0}$ and such that $D \subseteq D_{0} \subseteq J$. Then $L / K_{1}$ is purely inseparable with finite exponent $m$ so $B^{p^{m}} \subseteq J^{p^{m}} \subseteq K_{1}$. Let $\left\{\xi_{i}\right\}_{i=1}^{\infty}$ be a subset of $B$ and choose integers $n \geqslant 1, k_{0} \geqslant 1$, $m_{1} \geqslant 0$ and a nonzero element $d_{1}$ in $A\left[\xi_{1}^{p^{m_{1}}}, \ldots, \xi_{k_{0}}^{m_{1}}\right]$ as in (2.8) so that $d_{1} \xi_{k}^{m_{1}}$ is a polynomial in $A\left[\xi_{1}^{p_{1}}, \ldots, \xi_{k-1}^{p_{1}^{m_{1}}}\right] \subseteq D\left[\xi_{1}^{p_{1}}, \ldots, \xi_{k-1}^{p_{1}^{m_{1}}}\right]$ with total degree not more than $p^{m_{1}} \gamma_{k+n}$ in the $\xi_{i}^{p^{m_{1}}}$. If $m_{1}<m$ then $\left(d_{1} \xi_{k}^{p^{m_{1}}}\right)^{p^{m-m_{1}}}=d_{1}^{p^{m-m_{1}}} \xi_{k}^{p^{m}}$ is a polynomial in $D\left[\xi_{1}^{p^{m}}, \ldots, \xi_{k-1}^{p^{m}}\right]$ with total degree not more than $p^{m} \gamma_{k+n}$ in the $\xi_{i}^{p^{m}}$, so we may assume that $m_{1} \geqslant m$. Thus, $L^{p^{m_{1}}} \subseteq K_{1}$ and, in particular, $d_{1} \in K_{1}$. There exists a nonzero element $d_{2} \in D_{0}$ such that $d_{2} d_{1} \in D_{0}$ and $d_{2} d_{1} \xi_{i}^{p_{1}} \in D_{0}$ for $1 \leqslant i \leqslant k_{0}$. It follows that $d_{2} d_{1} \xi_{k}^{m_{1}} \in D_{0}\left[\xi_{1}^{p_{1}}, \ldots, \xi_{k-1}^{m_{1}}\right]$ for each $k>1$. Since $D_{0}$ is algebraic over $D, d_{2} d_{1} D_{0} \cap D \neq(0)$. If $d$ is any nonzero element of $d_{2} d_{1} D_{0} \cap D$ then $d$ meets the requirements stated in Theorem 2.1. This completes the proof of Theorem 2.1.

Corollary 2.10. Let $D_{0}$ be as in Theorem 2.1 and for each positive integer $k$ let $h_{k}$ denote the polynomial $1+x+\cdots+x^{k-1}$. For each subset $\left\{\xi_{i}\right\}_{i=1}^{\infty}$ of $B$ there exists a nonzero element $d$ in $D$ and nonnegative integers $m$ and $n$ such that $d^{h_{k}\left(p^{m} \eta_{k+n}\right)} \xi_{k}^{p^{m}} \in D_{0}$ for each $k$.

Proof. Applying Theorem 2.1 we get nonnegative integers $m$ and $n$ and a nonzero element $d$ in $D$ such that $d \xi_{k}^{p^{m}}$ is a polynomial in $D_{0}\left[\xi_{1}^{p^{m}}, \ldots, \xi_{k-1}^{p^{m}}\right]$ with degree at most $p^{m} \gamma_{k+n}$ in the $\xi_{i}$. In particular, $d \xi_{1}^{p^{m}}=d^{h_{1}\left(p^{m} \gamma_{1+n}\right)} \xi_{1}^{p^{m}} \in D_{0}$. Suppose

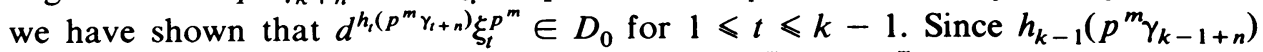

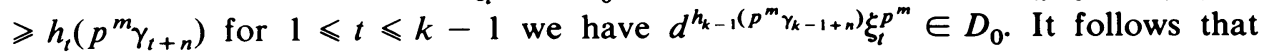

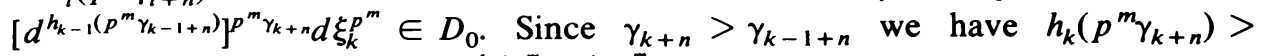
$p^{m} \gamma_{k+n} h_{k-1}\left(p_{k-1+n}^{m}\right)+1$, so $d^{h_{k}\left(p^{m} \gamma_{k+n}\right)} \xi_{k}^{p^{m}} \in D_{0}$.

Corollary 2.11. If $D_{0}$ is as in Theorem 2.1 then for each subset $\left\{\xi_{i}\right\}_{i=1}^{\infty}$ of $B$ there exists a nonzero element $d$ in $D$ and a sequence $\left\{n_{i}\right\}_{i=1}^{\infty}$ of positive integers such that $\left(d \xi_{i}\right)^{n_{i}} \in D_{0}$ for each $i$.

Proof. Applying Corollary 2.10 to the set $\left\{\xi_{k}^{h_{k}\left(p^{k} \gamma_{2 k}\right)}\right\}_{k=1}^{\infty}$ we get nonnegative integers $m$ and $n$ and a nonzero element $d_{1}$ in $D$ such that $d_{1}^{h_{k}\left(p^{m} \gamma_{k+n}\right)} \xi_{k}^{p^{m} h_{k}\left(p^{k} \gamma_{2 k}\right)} \in$ $D_{0}$ for each $k$. In particular, if $k \geqslant \max \{m, n\}$ then $\left(d_{1} \xi_{k}\right)^{p^{m} h_{k}\left(p^{k} \gamma_{2 k}\right)} \in D_{0}$. If $d_{2}$ is any nonzero element of $D$ such that $d_{2} \xi^{p^{m} h_{1}\left(p^{t} \gamma_{2}\right)} \in D_{0}$ for $1<t<\max \{m, n\}$ then Corollary 2.11 follows with $d=d_{1} d_{2}$ and $n_{k}=p^{m} h_{k}\left(p^{k} \gamma_{2 k}\right)$.

In some of our later results we shall wish to choose $D_{0}$ to meet certain conditions. Our next remark will allow us to do this. 
REMARK 2.12. Let $D$ and $J_{0}$ be integral domains with quotient fields $K$ and $K_{0}$, respectively, and suppose that $K_{0} / K$ is a finite separable extension. Then there exists $\theta \in K_{0}$ such that $K_{0}=K(\theta)$ and $D_{0}=D[\theta] \subseteq J_{0}$ is an integral extension of $D$. Further, if $D$ is integrally closed and $D^{\prime}$ is the integral closure of $D$ in $K_{0}$, there exists a nonzero element $d$ in $D$ such that $d D^{\prime} \subseteq D_{0}$. (Cf. the proof of Theorem 41.7 in [4].)

3. Domains in which (1)-(5) are equivalent. To show that (1)-(5) of (1.2) are equivalent for the integral domains $A$ and $B$, where $A \subset B$, it suffices, in view of Proposition 3.7, to show that $(5) \rightarrow(1)$. Our basic approach is to reduce to the case in which $B$ is an overring of $A$ (that is, $A$ and $B$ have the same quotient field) and apply the results of [1], as restated below, to show that (a) of (1.1) holds. In Lemma 3.4 it becomes necessary to pass to an overring $D$ of $A$ and show that if (e) holds for $A \subset B$ then (a) holds for $D \subset J$, where $J=D[B]$. Thus, as in Theorem 2.1, some of our preliminary results are stated in this more general setting. The following observation will be useful.

REMARK 3.1. Let $A, B, D$, and $J$ be integral domains such that $A \subset B \subseteq J$, $A \subseteq D \subseteq J$ and $J=D[B]$. In order to show that the domains $D$ and $J$ satisfy (1) of (1.2) [respectively, (a) of (1.1)] it suffices to show that (1) [respectively, (a)] holds for each subset $\left\{\xi_{i}\right\}_{i=1}^{\infty}$ of $B$.

Our next several results, (3.2)-(3.6), will facilitate the reduction to the case in which $B$ is an overring of $A$. In this case several instances are given in [1] in which conditions (a)-(d) of (1.1) are equivalent. The proofs given in [1], with slight alterations (particularly the application of Remark 3.1 and the substitution of Theorem 2.1, Corollary 2.10, and Corollary 2.11 with $D_{0}=D$, respectively, for Theorem 1.3, Lemma 1.4 and Lemma 1.5 of [1]), actually yield the more general results stated in Lemmas 3.2 and 3.3 below.

LeMMA 3.2. Let $A, B, D$, and $J$ be integral domains with quotient field $K$ such that $A \subset B \subseteq J, A \subseteq D \subseteq J$, and $J=D[B]$. One of the following holds:

(i) Condition (e) of (1.1) fails for the domains $A$ and $B$.

(ii) Condition (a) of (1.1) holds for the domains $D$ and J.

(iii) For each countable subset $S$ of nonzero elements of $D$ there exists a subset $\left\{\xi_{i}\right\}_{i=1}^{\infty}$ of $B$ such that if $d, m, n$ are as in Theorem 2.1 (with $D_{0}=D$ ) then divides no element of $S$.

Proof. Cf. Proposition 2.3 of [1, p. 186].

LEMMA 3.3. With notation as in Lemma 3.2, in each of the following cases if (e) of (1.1) holds for the domains $A$ and $B$ then (a) of (1.1) holds for the domains $D$ and $J$.

(1) $D$ is root closed in $J$. In this case we can take $m=0$ in (a) (cf. [1, Theorem 1.6]).

(2) The quotient field $K$ of $D$ is countably generated over $D$ (cf. [1, Theorem 2.4]).

(3) $D$ is Noetherian (cf. [1, Theorem 2.5]).

LemMa 3.4. Let $A$ be an integral domain with quotient field $K$. If $A$ has Krull dimension one then (a)-(e) of (1.1) are equivalent for each overring $B$ of $A$. 
Proof. Suppose that (e) holds and let $\left\{\xi_{i}\right\}_{i=1}^{\infty}$ be a subset of $B$. By Corollary 2.10 (with $A=D=D_{0}$ ) there exist a nonzero element $d$ in $A$, an integer $m \geqslant 0$, and positive integers $\left\{n_{i}\right\}_{i=1}^{\infty}$ such that $d^{n_{i}} \xi_{i}^{p^{m}} \in A$ for each $i$. Let $\left\{M_{\alpha}\right\}_{\alpha \in \Lambda}$ be the set of maximal ideals of $A$ such that $\left\{\xi_{i}^{p^{m}}\right\}_{i=1}^{\infty} \underline{Z} A_{M_{\alpha}}$. If no such maximal ideals exist then $\left\{\xi_{i}^{p^{m}}\right\}_{i=1}^{\infty} \subseteq A$ and (a) holds. If $\left\{M_{\beta}\right\}_{\beta \in \Gamma}$ is the set of maximal ideals of $A$ that contain $d$ then $\left\{M_{\beta}\right\}_{\beta \in \Gamma} \supseteq\left\{M_{\alpha}\right\}_{\alpha \in \Lambda}$. In partiuclar, if $M \notin\left\{M_{\beta}\right\}_{\beta \in \Gamma}$ then $\left\{\xi_{i}^{p^{m}}\right\}_{i=1}^{\infty} \subseteq A_{M}$. Set $S=A \backslash \cup_{\beta \in \Gamma} M_{\beta}$ and let $D=A_{S}$. If $M$ is a maximal ideal of $A$ such that $M \notin\left\{M_{\beta}\right\}_{\beta \in \Gamma}$ then $A=M+d A$ so there exist $m \in M$ and $a \in A$ such that $1=m+a d$. Thus, $m=1-a d \in M \cap S$ and $M D=D$. It follows that $\left\{M_{\beta} D\right\}_{\beta \in \Gamma}$ is the set of maximal ideals of $D$. But $d \in \cap_{\beta \in \Gamma} M_{\beta} D$ and $D$ has Krull dimension one, so $K=D[1 / d]$. By Lemma 3.3(2), (a) of (1.1) holds for the domains $D$ and $J=D[B]$. In particular, there exist a nonzero element $d_{1}$ in $D$ and a nonnegative integer $n$ such that $\left\{d_{1} \xi_{i}^{p^{n}}\right\} \subseteq D$. If $d_{2} \in d_{1} D \cap A$ and $k \geqslant$ $\max \{m, n\}$ then $\left\{d_{2} \xi_{i}^{p^{k}}\right\} \subseteq A$ so (a) holds for the domains $A$ and $B$.

REMARK 3.5. Let $D \subseteq D_{1} \subseteq J_{1} \subseteq J$ be integral domains such that $J_{1}$ is an overring of $D_{1}$ with quotient field containing $K_{0}$. If one of the conditions (1)-(5) of (1.2) holds for the domains $D$ and $J$ then the analogous condition from (a)-(e) of (1.1) holds for the domains $D_{1}$ and $J_{1}$. Further, we can take $d$ in $D^{*}$ in (a) and in (b) we can write $\left(D_{1}[[X]]\right)_{D_{i}^{*}}=\left(D_{1}[[X]]\right)_{D^{*}}$.

Proof. We show that $(2) \rightarrow(b)$, the other implications being obvious. Thus, let $D_{0}=D[\theta]$ be as in (2). Then $\left(J_{1}[[X]]\right)^{p^{m}} \subseteq\left(D_{0}[[X]]\right)_{D^{*}} \subseteq\left(D_{1}[\theta][[X]]\right)_{D^{*}}$. But $\theta$ is in the quotient field of $D_{1}$ and is integral over $D_{1}$. Since $D_{1}$ is algebraic over $D$ there exists $d \in D$ such that $d D_{1}[\theta] \subseteq D_{1}$. It follows that $\left(J_{1}[[X]]\right)^{p^{m}} \subseteq\left(D_{1}[[X]]\right)_{D^{*}}$.

LEMMA 3.6. Let $D$ and $J$ be integral domains such that $D \subseteq J$ and (5) of (1.2) holds and let $D_{0}=D[\theta]$ be an integral extension of $D$ with quotient field $K_{0}$. Set $J_{0}=J \cap K_{0}$ and let $\bar{D}$ be the integral closure of $D$ in $J_{0}$. The domains $D$ and $J$ satisfy (1) of (1.2) if and only if the domains $D_{0}$ and $\bar{D}$ satisfy (a) of (1.1).

Proof. Suppose that $D_{0} \subseteq \bar{D}$ satisfies (a) of (1.1) and let $\left\{\xi_{i}\right\}_{i=1}^{\infty}$ be a subset of $J$. By Theorem 2.1, $L$ has finite exponent over $K_{0}$ so there exists a positive integer $n$ such that $J^{p^{n}} \subseteq K_{0}$. If we set $\zeta_{i}=\xi_{i}^{p^{n}}$ for each $i$ then $\left\{\zeta_{i}\right\}_{i=1}^{\infty} \subseteq J_{0}$ and by Corollary 2.11 there exist a nonzero element $d_{1}$ in $D$ and a sequence $\left\{n_{i}\right\}_{i=1}^{\infty}$ of positive integers such that $\left(d_{1} \zeta_{i}\right)^{n_{i}} \in D_{0} \subseteq \bar{D}$ for each $i$. Thus, $\left\{d_{1} \zeta_{i}\right\}_{i=1}^{\infty}$ is a subset of $\bar{D}$. Since (a) holds for $D_{0} \subseteq \bar{D}$ there exist a nonnegative integer $m$ and a nonzero element $d_{2}$ in $D$ such that $d_{2}\left(d_{1} \zeta_{i}\right)^{p^{m}} \in D_{0}$ for each $i$. If $d=d_{2} d_{1}^{p^{m}}$ then $d \xi_{i}^{p^{m+n}} \in$ $D_{0}$ for each $i$. Now let $D_{1}$ be an integral domain such that $D \subseteq D_{1} \subseteq J$ and $D_{1}$ has quotient field containing $K_{0}$. Then $d \xi_{i}^{p^{m+n}} \in D_{0}=D[\theta] \subseteq D_{1}[\theta]$ for each $i$ and, as in the proof of Remark 3.5, there exists a nonzero element $d_{3}$ in $D$ such that $d_{3} D_{1}[\theta] \subseteq D_{1}$. Thus, $d_{3} d \xi^{p^{m+n}} \in D_{1}$ for each $i$ and (1) holds for $D$ and $J$. The converse is given in Remark 3.5.

Proposition 3.7. The following implications hold between conditions (1)-(5) of (1.2).

$$
(1) \leftrightarrow(2) \rightarrow(3) \rightarrow(4) \rightarrow(5) .
$$


Proof. The implications $(3) \rightarrow(4) \rightarrow(5)$ are evident and that $(1) \rightarrow(2)$ follows from Remark 2.12 and an adaptation of the proof of Proposition 2.1 of [1].

To show that $(2) \rightarrow(1)$ let $D_{0}=D[\theta]$ be as in (2) and let $\bar{D}$ be as in Lemma 3.6. By Remark 3.5 the domains $D_{0}$ and $\bar{D}$ satisfy (b) of (1.1). But (b) $\rightarrow$ (a) [1, Proposition 2.1] so by Lemma 3.6 the domains $D$ and $J$ satisfy (1).

To see that $(2) \rightarrow(3)$ let $D_{0}=D[\theta]$ be as in (2). By [3, Lemma 2.5] $D_{0}[[X]]$ is integral over $D[[X]]$ and, clearly, $J[[X]]$ is algebraic over $D_{0}[[X]]$. Thus, $J[[X]]$ is algebraic over $D_{0}[[X]]$.

Let $K$ and $L$ be fields with $K \subseteq L$. In [3] Gilmer has determined necessary and sufficient conditions in order that $L\left[\left[X_{1}, \ldots, X_{n}\right]\right]$ be integral over $K\left[\left[X_{1}, \ldots, X_{n}\right]\right]$. The main results of [3] are essentially summarized in [3, Corollary 4.2] and may be restated as follows.

(3.8) $L\left[\left[X_{1}, \ldots, X_{n}\right]\right]$ is integral over $K\left[\left[X_{1}, \ldots, X_{n}\right]\right]$ if and only if $L$ has finite exponent over $K_{0}$ and $\left[K_{0}: K\right]$ is finite, where $K_{0}$ is the maximal separable extension of $K$ in $L$.

Our next result, Theorem 3.9, shows that (1)-(5) of (1.2) are equivalent for Noetherian domains and extends (3.8) to Noetherian domains.

THEOREM 3.9. If $D$ is a Noetherian integral domain and $J$ is an integral domain containing $D$ then each of the following statements is equivalent to (1)-(5) of (1.2).

(i) There exists a nonnegative integer $m$ and a finite integral extension $D_{1}$ of $D$ such that $D \subseteq D_{1} \subseteq J, D_{1}$ has quotient field $K_{0}$, and $J^{p^{m}} \subseteq D_{1}$.

(ii) If $Y_{\Delta}=\left\{y_{i}\right\}_{i \in \Delta}$ is any set of analytic indeterminates over $D$ then $J\left[\left[Y_{\Delta}\right]\right]$ is integral over $D\left[\left[Y_{\Delta}\right]\right]$.

(iii) $J[[X]]$ is integral over $D[[X]]$.

Proof. (i) $\rightarrow$ (ii). If $J^{p^{m}} \subseteq D_{1}$ then clearly $J\left[\left[Y_{\Delta}\right]\right]$ is integral over $D_{1}\left[\left[Y_{\Delta}\right]\right]$. But $D_{1}$ is a finite $D$-module so $D_{1}\left[\left[Y_{\Delta}\right]\right]$ is a finite $D\left[\left[Y_{\Delta}\right]\right]$-module (cf. [3, Lemma 2.5]). In particular, $D_{1}\left[\left[Y_{\Delta}\right]\right]$ is integral over $D\left[\left[Y_{\Delta}\right]\right]$ and, hence, $J\left[\left[Y_{\Delta}\right]\right]$ is integral over $D\left[\left[Y_{\Delta}\right]\right]$.

That (ii) $\rightarrow$ (iii) is clear and (iii) $\rightarrow(3) \rightarrow(4) \rightarrow(5)$.

$(5) \rightarrow(1)$. By Theorem 2.1 and Remark 2.12 there exists an integral extension $D_{0}=D[\theta]$ of $D$ with quotient field $K_{0}$ such that $D \subseteq D_{0} \subseteq J$. With notation as in Lemma 3.6, the domains $D_{0}$ and $\bar{D}$ satisfy (e) of (1.1). But $D_{0}$ is Noetherian so by Lemma $3.3, D_{0}$ and $\bar{D}$ satisfy (a) of (1.1). It follows from Lemma 3.6 that the domains $D$ and $J$ satisfy (1) of (1.2). In particular, it follows from Proposition 3.7 that (1)-(5) are equivalent.

(2) $\rightarrow$ (i). Let $D_{0}$ and $m$ be as in (2). Thus, $D_{0}$ has quotient field $K_{0}$ and $J^{p^{m}} \subseteq K_{0}$. If we set $D_{1}=D_{0}\left[J^{p^{m}}\right]$ then $D \subseteq D_{1} \subseteq J, D_{1}$ has quotient field $K_{0}$, and $J^{p^{m}} \subseteq D_{1}$. To show that (i) holds it suffices to show that $D_{1}$ is finitely generated as a $D$-module. Thus, let $\left\{\lambda_{i}\right\}_{i=1}^{\infty}$ be a subset of $D_{1}$ and let $M$ be the $D$-module generated by $\left\{\lambda_{i}\right\}_{i=1}^{\infty}$. By (2) there exists a nonzero element $d$ in $D$ such that $\left\{d \lambda_{i}\right\}_{i=1}^{\infty} \subseteq D_{0}$. Thus, $d M$ is a submodule of the Noetherian $D$-module $D_{0}$ and, hence, $d M$ is finitely generated. It follows that $M$, and therefore $D_{1}$, is finitely generated as a $D$-module. 
THEOREM 3.10. If $D$ is an integrally closed integral domain then (1)-(5) of (1.2) are equivalent.

Proof. Let $J$ be an integral domain that contains $D$ and suppose that (5) of (1.2) holds for $D$ and $J$. It follows from Theorem 2.1 and Remark 2.12 that there exists a finite integral extension $D_{0}=D[\theta]$ of $D$ with quotient field $K_{0}$ such that $D \subseteq D_{0}$ $\subseteq J$. Further, if $D^{\prime}$ is the integral closure of $D$ in $K_{0}$ then, as noted in Remark 2.12, there exists a nonzero element $d$ in $D$ such that $d D^{\prime} \subseteq D_{0}$. If $\bar{D}$ is the integral closure of $D$ in $J_{0}=J \cap K_{0}$, then $\bar{D} \subseteq D^{\prime}$ so $d \bar{D} \subseteq D_{0}$. Therefore the domains $D_{0}$ and $\bar{D}$ satisfy (a) of (1.1) and by Lemma 3.6 the domains $D$ and $J$ satisfy (1) of (1.2).

THEOREM 3.11. If the quotient field $K$ of $D$ is countably generated over $D$ then (1)-(5) of (1.2) are equivalent.

Proof. Suppose that $D \subset J$ and that (5) of (1.2) holds. If $D_{0}$ and $\bar{D}$ are as in the proof of Theorem 3.10 above then $D_{0}$ and $\bar{D}$ satisfy (e) of (1.1) by Remark 3.5. Moreover, since $K_{0}=\left(D_{0}\right)_{D^{*}}, K_{0}$ is countably generated over $D_{0}$. By Lemma 3.3, (a) of (1.1) holds for $D_{0}$ and $\bar{D}$ so by Lemma 3.6, (1) of (1.2) holds for $D$ and $J$.

THEOREM 3.12. If D has Krull dimension one then (1)-(5) of (1.2) are equivalent.

Proof. Let $J, D_{0}$ and $\bar{D}$ be as in the preceding two proofs. Then $D_{0}$ and $\bar{D}$ satisfy (e) of (1.1) and $D_{0}$ has Krull dimension one. The theorem follows from Lemmas 3.4 and 3.6.

The four previous theorems have established sufficient conditions on $D$ in order that (1)-(5) of (1.2) be equivalent. Our next theorem, Theorem 3.14, will give sufficient conditions on $J$. We first prove the following result.

Proposition 3.13. Let $D, D_{0}$ and $J$ be as in Theorem 2.1. For each subset $\left\{\zeta_{i}\right\}_{i=1}^{\infty}$ of $J$ there exists a nonzero element $d$ in $D$ such that $d \in \cap_{i=1}^{\infty} \zeta_{i}^{-1} D_{0}\left[\zeta_{1}^{-1}, \ldots, \zeta_{i}^{-1}\right]$.

Proof. Suppose that $J^{p^{m_{1}}} \subseteq K_{0}$ and let $\delta_{i}=\zeta_{i}^{p^{m_{1}}}$ for each $i$. Set $\xi_{1}=\delta_{1}$ and for $k>1$ set $\xi_{k}=\delta_{k}\left(\xi_{1} \cdots \xi_{k-1}\right)^{p^{k} \gamma_{2 k}}$ where $\left\{\gamma_{i}\right\}_{i=1}^{\infty}$ is as in Theorem 2.1. By Theorem 2.1 there exist integers $n \geqslant 1, m \geqslant 0$ and a nonzero element $d_{1}$ in $D$ such that, for each $k \geqslant 1, d_{1} \xi_{k}^{p^{m}}=g_{k}\left(\xi_{1}^{p^{m}}, \ldots, \xi_{k-1}^{p^{m}}\right) \in D_{0}\left[\xi_{1}^{p^{m}}, \ldots, \xi_{k-1}^{p^{m}}\right]$ with total degree at $\operatorname{most} p^{m} \gamma_{k+n}$ in $\xi_{1}^{p^{m}}, \ldots, \xi_{k-1}^{p^{m}}$. If $k \geqslant k_{0}=\max \{m, n\}$ then

$$
\begin{aligned}
d_{1} \delta_{k}^{p^{m}} & =d_{1} \xi_{k}^{p^{m}}\left(\xi_{1} \cdots \xi_{k-1}\right)^{-p^{k+m} \gamma_{2 k}} \\
& =\left(\xi_{1}^{p^{m}} \cdots \xi_{k-1}^{p^{m}}\right)^{-p^{k} \gamma_{2 k}} g_{k}\left(\xi_{1}^{p^{m}}, \ldots, \xi_{k-1}^{p^{m}}\right) \in D_{0}\left[\xi_{1}^{-p^{m}}, \ldots, \xi_{k-1}^{-p^{m}}\right] .
\end{aligned}
$$

Since $\left\{\delta_{i}^{p^{m}}\right\}$ is contained in the quotient field of $D_{0}$ and since $\left(D_{0}\right)_{D^{*}}$ is the quotient field of $D_{0}$, there exists a nonzero element $d_{2}$ in $D$ such that $d_{2} \delta_{i}^{p^{m}} \in D_{0}$ for $1 \leqslant i \leqslant k_{0}$. If we set $d=d_{1} d_{2}$ then $d \in \delta_{i}^{-p^{m}} D_{0}\left[\xi_{1}^{-p^{m}}, \ldots, \xi_{i-1}^{-p}\right] \subseteq$ $\zeta_{i}^{-1} D_{0}\left[\zeta_{1}^{-1}, \ldots, \zeta_{i}^{-1}\right]$ for each $i$.

THEOREM 3.14 (CF. [1, THEOREM 1.10]). Let $J=\left(D_{1}\right)_{S}$ where $D_{1}$ is a finite integral extension of $D$ and $S$ is a multiplicative system in $D_{1}$. Then (1)-(5) of (1.2) are equivalent to the condition that for each subset $\left\{s_{i}\right\}_{i=1}^{\infty}$ of $S$ there exists a nonzero element $d$ in $D$ such that $d \in \cap_{i=1}^{\infty} s_{i} D_{1}$. 
Proof. Suppose that (5) of (1.2) holds for the domains $D$ and $J=\left(D_{1}\right)_{S}$ and let $\left\{s_{i}\right\}_{i=1}^{\infty}$ be a subset of $S$. Since, in the notation of Theorem 2.1, $L$ is the quotient field of $D_{1}$, the domains $D, D_{1}$, and $J$ satisfy the hypotheses of Proposition 3.13. If we take $\zeta_{i}=1 / s_{i}$ it follows from Proposition 3.13 that there exists a nonzero element $d$ in $D$ such that $d \in \cap_{i=1}^{\infty} s_{i} D_{1}$. To see that this condition implies (1) of (1.2) let $D_{0}$ be any integral domain such that $D \subseteq D_{0} \subseteq J$ and $D_{0}$ has quotient field containing $K_{0}$ and let $\left\{\xi_{i}\right\}_{i=1}^{\infty}$ be a subset of $J=\left(D_{1}\right)_{s}$. Choose $s_{i} \in S$ so that $s_{i} \xi_{i} \in D_{1}$ and let $d$ be a nonzero element of $D$ such that $d \in \cap_{i=1}^{\infty} s_{i} D_{1}$. Then $\left\{d \xi_{i}\right\}_{i=1}^{\infty} \subseteq D_{1}$. Suppose that $D_{1}=D\left[\lambda_{1}, \ldots, \lambda_{n}\right]$ where each $\lambda_{i}$ is integral over $D$. Since $[L: K]$ is finite by assumption, $L$ has finite exponent over $K_{0}$. Thus, there exists an integer $m \geqslant 0$ such that $\left\{\lambda_{i}^{p^{m}}\right\}_{i=1}^{n} \subseteq K_{0}$. Therefore, $\left\{d^{p^{m}} \xi_{i}^{p^{m}}\right\}_{i=1}^{\infty} \subseteq D_{1}^{p^{m}} \subseteq$ $D\left[\lambda_{1}^{p^{m}}, \ldots, \lambda_{n}^{p^{m}}\right] \subseteq D_{0}\left[\lambda_{1}^{p^{m}}, \ldots, \lambda_{n}^{p^{m}}\right]$. But there exists $d_{1} \in D^{*}$ such that $d_{1} D_{0}\left[\lambda_{1}^{p^{m}}, \ldots, \lambda_{n}^{p^{m}}\right] \subseteq D_{0}$ so if $d_{2}=d_{1} d^{p^{m}}$ then $\left\{d_{2} \xi_{i}^{p^{m}}\right\}_{i=1}^{\infty} \subseteq D_{0}$ and (1) holds.

Proposition 3.15. Let $A, B$, and $C$ be integral domains with quotient fields $F, K$, and $L$, respectively, such that $A \subset B \subset C$. If conditions (1)-(5) of (1.2) are equivalent for the domains $A$ and $B$ and for the domains $B$ and $C$, then they are equivalent for the domains $A$ and $C$.

Proof. Suppose that (5) holds for the domains $A$ and $C$ and let $F_{0}$ be the maximal, separable extension of $F$ in $L$. By Theorem $2.1 L$ is algebraic over $F,\left[F_{0}\right.$ : $F]$ is finite, and $L$ has finite exponent over $F_{0}$. To show that (1) holds let $A_{0}$ be an integral domain with quotient field containing $F_{0}$ such that $A \subseteq A_{0} \subseteq C$ and let $\left\{\xi_{i}\right\}_{i=1}^{\infty}$ be a subset of $C$. Let $K_{0}$ be the maximal separable extension of $K$ in $L$ and set $B_{0}=B\left[A_{0}\right]$. Clearly, $L$ is purely inseparable over the quotient field of $B_{0}$ (since it contains $F_{0}$ ), so $K_{0}$ is contained in the quotient field of $B_{0}$. But (5) also holds for the domains $B$ and $C$ and (1)-(5) of (1.2) are equivalent for these domains, so there exist a nonzero element $b$ in $B$ and an integer $m_{1} \geqslant 0$ such that $\left\{b \xi_{i}^{p^{m_{1}}}\right\} \subseteq B_{0}=$ $B\left[A_{0}\right]$ for each $i$. For each $i$, write $b \xi_{i}^{p^{m_{1}}}=\sum_{j=1}^{k_{i}} b_{i j} \alpha_{i j}$ where $b_{i j} \in B$ and $\alpha_{i j} \in A_{0}$. If $F_{1}$ is the maximal separable extension of $F$ in $K$ then $F_{1}=F_{0} \cap K$, so $A_{0} \cap B$ has quotient field containing $F_{1}$. Since (5) $\rightarrow(1)$ for the domains $A$ and $B$, there exist a nonzero element $a_{1}$ in $A$ and an integer $m_{2} \geqslant 0$ such that $\left\{a_{1} b_{i j}^{p^{m_{2}}}\right\} \subseteq A_{0} \cap B \subseteq$ $A_{0}$. Thus, $a_{1} b^{p^{m_{2}}} \xi_{i}^{p^{m_{1}+m_{2}}}=\sum_{j=1}^{k_{i}} a_{1} b_{i j}^{p^{m_{2}}} \alpha_{i j}^{p^{m_{2}}} \in A_{0}$ for each $i$. If $a$ is any nonzero element in $\left(a_{1} b^{p^{m_{2}}}\right) B \cap A$ and if $m=m_{1}+m_{2}$ then $\left\{a \xi_{i}^{p^{m}}\right\}_{i=1}^{\infty} \subseteq A_{0}$ and (1) holds.

Corollary 3.16. Let $D$ and $J$ be integral domains with quotient fields $K$ and $L$, respectively, and suppose that $D \subset J$. Set $T=J \cap K$ and suppose that $D$ is root closed in $T$ or $T$ is a quotient overring of $D$. Then in each of the following cases (1)-(5) are equivalent for the domains $D$ and $J$.

(1) $T$ is Noetherian.

(2) $T$ is integrally closed.

(3) $K$ is countably generated over $T$.

(4) $T$ has Krull dimension one.

(5) $J=\left(T_{1}\right)_{S}$ where $T_{1}$ is a finite integral extension of $T$ and $S$ is a multiplicative system in $T_{1}$. 
Proof. The result is an immediate consequence of Proposition 3.15, Lemma 3.3(1), and Theorems 3.9, 3.10, 3.11, 3.12, and 3.14.

We conclude by proving the equivalence of stronger versions of conditions (3)-(5) of (1.2).

TheOREM 3.17. If $D$ and $J$ are integral domains such that $D \subset J$ then the following statements are equivalent.

(1) $J\left[\left[X_{1}, \ldots, X_{n}\right]\right]$ is algebraic over $D\left[\left[X_{1}, \ldots, X_{n}\right]\right]$ for each positive integer $n$.

(2) $J\left[\left[X_{1}, \ldots, X_{n}\right]\right]$ has finite transcendence degree over $D\left[\left[X_{1}, \ldots, X_{n}\right]\right]$ for each positive integer $n$.

(3) For each positive integer $n$, if $R$ is an integral domain such that $D\left[\left[X_{1}, \ldots, X_{n}\right]\right] \subset R \subseteq J\left[\left[X_{1}, \ldots, X_{n}\right]\right]$ then $R \nRightarrow D\left[\left[X_{1}, \ldots, X_{n}\right]\right]\left[\left[Y_{\omega}\right]\right]$ via a $D\left[\left[X_{1}, \ldots, X_{n}\right]\right]$-isomorphism.

Proof. It suffices to show that (3) $\rightarrow(1)$. Thus if $n$ is any positive integer and $R$ is an integral domain such that $D\left[\left[X_{1}, \ldots, X_{n}\right]\right]\left[\left[X_{n+1}\right]\right] \subset R \subseteq$ $J\left[\left[X_{1}, \ldots, X_{n}\right]\right]\left[\left[X_{n+1}\right]\right]$ then $R \nRightarrow D\left[\left[X_{1}, \ldots, X_{n+1}\right]\right]\left[\left[Y_{\omega}\right]\right]$ via a $D\left[\left[X_{1}, \ldots, X_{n+1}\right]\right]$-isomorphism. By Theorem 2.1, if $L_{1}$ and $K_{1}$ are the quotient fields of $J\left[\left[X_{1}, \ldots, X_{n}\right]\right]$ and $D\left[\left[X_{1}, \ldots, X_{n}\right]\right]$, respectively, then $L_{1}$ is algebraic over $K_{1}$. In particular, $J\left[\left[X_{1}, \ldots, X_{n}\right]\right]$ is algebraic over $D\left[\left[X_{1}, \ldots, X_{n}\right]\right]$.

\section{REFERENCES}

1. J. T. Arnold and D. W. Boyd, Transcendence degree in power series rings, J. Algebra 57 (1979), 180-195.

2. R. Gilmer, $A$ note on the quotient field of $D[[X]]$, Proc. Amer. Math. Soc. 118 (1967), 1138-1140.

3. __ Integral dependence in power series rings, J. Algebra 11 (1969), 488-502.

4. __ Multiplicative ideal theory, Dekker, New York, 1972.

5. P. B. Sheldon, How changing $D[[X]]$ changes its quotient field, Trans. Amer. Math. Soc. 122 (1966), 321-333.

6. B. L. Van der Waerden, Modern algebra. I, Ungar, New York, 1949.

Department of Mathematics, Virginia Polytechnic Institute and State University, BlacksBURG, VIRGINIA 24061 